\title{
On the Processing of Very High-Resolution Spaceborne SAR Data
}

\author{
Pau Prats-Iraola, Senior Member, IEEE, Rolf Scheiber, Marc Rodriguez-Cassola, Josef Mittermayer, \\ Steffen Wollstadt, Francesco De Zan, Benjamin Bräutigam, Marco Schwerdt, Andreas Reigber, Senior \\ Member, IEEE, Alberto Moreira, Fellow Member, IEEE
}

\begin{abstract}
This paper addresses several important aspects that need to be considered for the processing of spaceborne SAR data with resolutions in the decimeter range. In particular, it will be shown how the motion of the satellite during the transmission/reception of the chirp signal and the effect of the troposphere deteriorate the impulse response function if not properly considered. Further aspects that have been investigated include the curved orbit, the array pattern for electronically steered antennas, and several considerations within the processing itself. For each aspect a solution is proposed, and the complete focusing methodology is expounded and validated using simulated point targets and staring spotlight data acquired by TerraSAR-X with $16 \mathrm{~cm}$ azimuth resolution and $300 \mathrm{MHz}$ range bandwidth.
\end{abstract}

Index Terms-Synthetic aperture radar (SAR), Spotlight SAR, SAR processing, stop-and-go approximation, troposphere.

\section{INTRODUCTION}

Spaceborne SAR processing is already an established topic. Many efficient solutions have been proposed over the last decades for every imaging mode, be it stripmap, spotlight, ScanSAR or TOPS (Terrain Observation by Progressive Scans). However, the assumptions of some of these algorithms start to be invalid depending on the acquisition geometry, especially in terms of the image resolution. While airborne SAR processors achieve nowadays resolutions in the decimeter range, the same approaches do not perform perfectly in a low Earth orbit (LEO) scenario, mainly due to the much greater sensor velocity and the larger distances involved. Furthermore, when getting close to decimeter resolutions several effects appear, which do not show up in an airborne scenario and must be taken into account to achieve a satisfactory focusing performance.

In order to design efficient processors that overcome these effects it is of great help to have real data available, which in the current case are provided by the TerraSAR-X (TSX) satellite. The TerraSAR-X mission represents one of the most successful high-resolution spaceborne SAR missions up to date, not only because of the impressive geometric and radiometric accuracy of the calibrated products [1], [2], but

Manuscript received May 8, 2013; revised August 17, 2013, October 16, 2013, and November 7, 2013; accepted December 3, 2013. TerraSAR-X is supported in part by the German Federal Ministry for Economics and Technology and realized in a public-private partnership between the German Aerospace Center (DLR) and Astrium GmbH.

The authors are with the Microwaves and Radar Institute, German Aerospace Center (DLR), 82234 Wessling, Germany (e-mail: Pau.Prats@dlr.de).

Color versions of one or more of the figures in this paper are available online at http://ieeexplore.ieee.org. also because the TSX satellite has become the perfect testbed for future SAR mission concepts thanks to its flexible commanding and operation. TSX has demonstrated for the first time in space several innovative modes like the TOPS mode [3]-[5], spaceborne bistatic imaging (together with its twin satellite, TanDEM-X) [6], bidirectional SAR imaging [7], or digital beamforming [8]. One of the last milestones achieved with the TSX satellite has been the acquisition of staring spotlight (ST) data. By exploiting the maximum azimuth steering capability of the sensor, it is possible to achieve a steering range of \pm 2.2 degrees, hence achieving a potential unweighted azimuth resolution of $16 \mathrm{~cm}$ [9]-[11]. Thanks to these data several effects could be precisely investigated and corrected, as expounded in the following sections.

The paper is divided as follows. Section II addresses the main aspects in the processing of very high resolution spotlight data, while Section III considers further secondary aspects. In all cases, a solution is proposed in order to achieve proper focusing. Section IV suggests a processing flow including the proposed corrections and finally Section $\mathrm{V}$ presents several results including simulations and high resolution spotlight data acquired by TSX.

TABLE I

COMPARISON OF THE TSX SPOTLIGHT MODES. VALUES GIVEN FOR SINGLE POLARIZATION.

\begin{tabular}{ccc}
\hline Mode & Az. resolution & Az. scene size \\
\hline Sliding spot. (SL) & $1.7 \mathrm{~m}$ & $10 \mathrm{~km}$ \\
High-res. spot. (HS) & $1.1 \mathrm{~m}$ & $5 \mathrm{~km}$ \\
Staring spot. (ST) & $0.21 \mathrm{~m}$ & $3 \mathrm{~km}-5 \mathrm{~km}$ \\
\hline
\end{tabular}

\section{Key Aspects of High Resolution Spaceborne} SAR PROCESSING

This section expounds the main effects that need to be considered when processing very high resolution spaceborne data. It is assumed that the high azimuth resolution is achieved by using the spotlight mode, be it staring or sliding [9]. The ST mode illuminates the same spot on ground during the whole integration time, while the sliding spotlight mode slides the beam to extend the azimuth coverage, which occurs at the expense of azimuth resolution. In both cases, the antenna is steered in the azimuth dimension, either mechanically or electronically. Table I summarizes the azimuth resolution and the azimuth scene extension for the three spotlight modes offered by the TSX satellite, where note that the ST mode has been recently implemented in the TSX ground segment 
TABLE II

TSX STARING SPOTLIGHT ACQUISITION OVER OBERPFAFFENHOFEN, GERMANY

\begin{tabular}{lc}
\hline Central frequency & $9.65 \mathrm{GHz}$ \\
Total azimuth processed bandwidth & $38.5 \mathrm{kHz}$ \\
Azimuth resolution & $16 \mathrm{~cm}$ \\
Chirp bandwidth & $300 \mathrm{MHz}$ \\
Ground range scene size & $8.8 \mathrm{~km}$ \\
Azimuth scene size & $3.4 \mathrm{~km}$ \\
Incidence angle & $35^{\circ}$ \\
Central point (longitude/latitude) & $11.264^{\circ}, 48.088^{\circ}$ \\
\hline
\end{tabular}

workflow and is available since October 2013. Due to the larger integration time of the ST mode, several effects appear, which can be neglected in the operational sliding spotlight (SL) and high resolution spotlight (HS) modes.

The first of these effects occurs due to the motion of the satellite during the transmission and reception of the chirp signal. This effect is well-known in the frame of frequencymodulated continuous-wave (FMCW) SAR radars mounted on airborne platforms [12], [13]. In Section II-A, the impact on high-resolution pulsed radars is analyzed.

The curved orbit and the validity of the hyperbolic approximation is also a topic that has been addressed before in the literature [14]-[16]. Section II-B suggests an innovative correction of the orbit curvature based on state-ofthe-art airborne SAR motion compensation techniques [17][19]. Besides being very simple to implement, it can also accommodate to a large extent the azimuth variance of the geometry.

One last aspect is the delay introduced by the troposphere in the range history. This delay can change significantly due to the variation of the squint (or azimuth) angle during the formation of the synthetic aperture, hence resulting in azimuth defocusing if not considered, especially at higher frequency bands. Section II-C addresses this topic and suggests a modelbased correction.

Through the different sections, ST data acquired by TSX are used. The particular data take was acquired over Oberpfaffenhofen, Germany, where five corner reflectors (CRs) were deployed in order to analyze their impulse response function (IRF) and validate the methodology. The main data take parameters appear in Table II.

\section{A. Stop-and-Go Approximation}

In the processing of spaceborne SAR raw data it is usually assumed that the platform does not move during the transmission of the pulse signal and the reception of the backscattered echoes. Such an assumption is usually called the stop-andgo or start-stop approximation and has mainly two effects. A "slow-time" one, which is linked to the fact that the satellite indeed moved between transmission and reception, e.g., about $30 \mathrm{~m}$ in the TSX case. This fact implies mainly a rangedependent azimuth shift of the focused signal, which can be efficiently considered with a linear azimuth phase ramp in the range-Doppler domain after the range cell migration correction (RCMC) given by [20]

$$
H_{\mathrm{SS}}^{\text {slow }}\left(f_{\mathrm{a}}, r\right)=\exp \left[\mathrm{j} \cdot 2 \pi \cdot \frac{r}{\mathrm{c}} \cdot f_{\mathrm{a}}\right],
$$

where $r$ is the range vector, $f_{\mathrm{a}}$ is the azimuth-frequency vector, and $\mathrm{c}$ is the speed of light. With this correction the targets have a constant azimuth time over range and are aligned with the annotated GPS instrument time. An additional effect occurs due to the slight bistatic operation, which becomes more evident the larger the integration time, i.e., the range history is the sum of two hyperbolas rather than just one. Although the effect is minor, it is properly accounted for in the correction suggested in the next section, where the accommodation of the curved orbit uses the position of the satellite at the transmission and reception events for the reference range.

A second effect deals with the motion of the satellite during the transmission and reception of the chirp signal itself, i.e., a "fast-time" effect or, in other words, a real Doppler effect. The TSX chirp signal has a length of about $50 \mu \mathrm{s}$, during which the satellite moves about $40 \mathrm{~cm}$ in the azimuth direction. This effect is well-known in FMCW SAR systems [12], [13]. The phenomenon was also discussed in [21] for pulsed radars, where the analysis was done in terms of a mismatch during the matched filtering in range. As shown in [21], the difference in the instantaneous frequency between the nominal range variation and the one including the motion of the platform is given by

$$
\Delta f=-\frac{2}{\lambda} \cdot \frac{\partial R(t)}{\partial t}=f_{\mathrm{DC}},
$$

where $\lambda$ is the wavelength, $R(t)$ is the range history and $f_{\mathrm{DC}}$ is the Doppler centroid caused by the platform movement during transmission/reception. By assuming a large time-bandwidth product, the shift in the signal in the range-time dimension is then given by

$$
\Delta \tau=\frac{\Delta f}{K_{\mathrm{r}}}=\frac{f_{\mathrm{DC}}}{K_{\mathrm{r}}},
$$

where $K_{\mathrm{r}}$ is the chirp rate and $\tau$ represents the fast time. This means that there is a range shift present in the signal as a function of the instantaneous azimuth frequency. In the case of large azimuth bandwidths, this effect will introduce different range shifts along the azimuth integration interval, hence deteriorating the impulse response function in both dimensions.

Another intuitive way to visualize this effect is by considering different azimuth phase ramps as a function of the range frequency, since, due to the large time-bandwidth product, there is a direct mapping between fast-time and range frequency. In other words, due to the motion of the satellite, the zero-Doppler time changes as a function of the range frequency, corresponding to a range-frequency dependent linear phase ramp in the azimuth dimension. The azimuth shift can then be expressed as

$$
\Delta t=\frac{\tau_{\mathrm{p}} \cdot f_{\mathrm{r}}}{B_{\mathrm{rg}}}=\frac{f_{\mathrm{r}}}{K_{\mathrm{r}}}
$$

where $f_{\mathrm{r}}$ is the range frequency, $t$ is the slow time and $\tau_{\mathrm{p}}$ and $B_{\mathrm{rg}}$ are the chirp length and chirp bandwidth, respectively.

Fig. 1 shows the resulting phase error due to the stop-andgo approximation measured over a corner reflector of the TSX ST acquisition and the corresponding IRF. The first plot on the left is the phase of the 2D phase spectrum after removing the global linear ramps due to the target position. The error reaches 

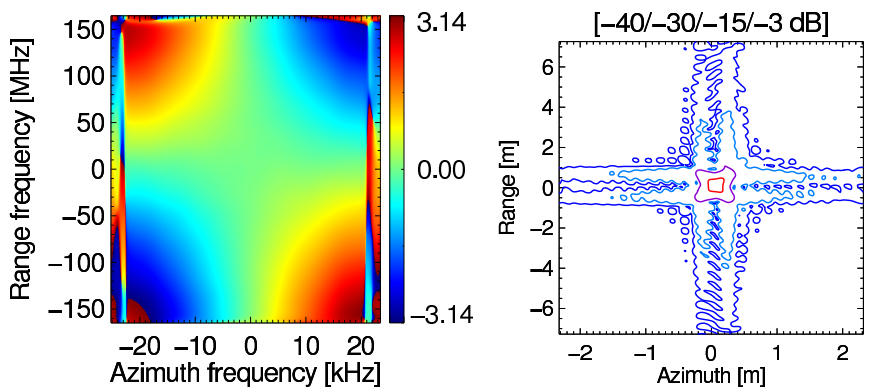

Fig. 1. Effect of the stop-and-go approximation in the TerraSAR-X staring spotlight mode. (Left) Residual phase in radians of a CR in the $2 \mathrm{D}$ frequency domain, and (right) the corresponding contour plot of the oversampled IRF. The chirp length is $50 \mu \mathrm{s}$, the bandwidth $300 \mathrm{MHz}$, and the azimuth processed bandwidth $38.5 \mathrm{kHz}$.

TABLE III

\begin{tabular}{lc} 
TERRASAR-X-LIKE ORBIT PARAMETERS \\
\hline Eccentricity & 0.001 \\
Inclination & $97.44^{\circ}$ \\
Semi-major axis & $6883.513 \mathrm{~km}$ \\
Argument of perigee & $90^{\circ}$ \\
Ascending node & $88.617^{\circ}$ \\
\hline
\end{tabular}

$\pm 177^{\circ}$ at the edges of the spectrum. If not corrected, this error introduces resolution loss of up to $15 \%$ in both dimensions for an azimuth bandwidth of $38.5 \mathrm{kHz}(16 \mathrm{~cm}$ resolution without weighting), a range bandwidth of $300 \mathrm{MHz}$ and a chirp length of $50 \mu \mathrm{s}$. Please note that in order to generate the plots of Fig. 1 and properly appreciate the effect of the fast-time stopand-go approximation, the suggested corrections for the other aspects, namely the consideration of the curved orbit and the troposphere, were applied (see Sections II-B and II-C, respectively).

Since the effect is space invariant, it can be easily corrected in the 2D frequency domain using the following phase function [12], [21]

$$
H_{\mathrm{ss}}^{\mathrm{fast}}\left(f_{\mathrm{a}}, f_{\mathrm{r}}\right)=\exp \left[\mathrm{j} \cdot 2 \pi \cdot \frac{f_{\mathrm{a}}}{K_{\mathrm{r}}} \cdot f_{\mathrm{r}}\right] .
$$

This phase correction shall be applied at the beginning of the processing before calling the focusing kernel. In the spotlight modes one needs to consider the azimuth aliasing at raw data level, which can be done either by using sub-apertures [4], [22], [23] or a deramping approach [24] (see Section IV).

\section{B. The Curved Orbit}

A hyperbolic range history, i.e., a linear track, is assumed by most spaceborne SAR image formation algorithms, but this approximation becomes less accurate the larger the integration time. For example, the TSX ST mode has an integration time of about 7 seconds, which yields the error that can be observed in Fig. 2 for the Oberpfaffenhofen scene. Such an error degrades the azimuth resolution and introduces asymmetric sidelobes.

Some solutions exist in the literature that use a numerical approach to circumvent this problem, e.g. [14], [15]. Also in the present case a numerical approach has been selected, which resembles the motion compensation approach in airborne SAR systems [17]-[19]. The line-of-sight (LOS) approximation error, $\delta r_{\text {hyp }}$, shown in Fig. 2, can be computed for a reference target in the middle of the scene, yielding

$$
\begin{aligned}
\delta r_{\mathrm{hyp}}\left(t ; r_{\mathrm{ref}}\right)= & \frac{1}{2} \cdot\left[\left|\mathbf{p}_{\mathrm{sat}}^{\mathrm{Tx}}(t)-\mathbf{p}_{\mathrm{bc}}\left(t ; r_{\mathrm{ref}}\right)\right|\right. \\
& \left.+\left|\mathbf{p}_{\mathrm{sat}}^{\mathrm{Rx}}(t)-\mathbf{p}_{\mathrm{bc}}\left(t ; r_{\mathrm{ref}}\right)\right|\right] \\
& -\sqrt{r_{\mathrm{ref}}^{2}+v_{\mathrm{e}, \mathrm{ref}}^{2}\left(t ; r_{\mathrm{ref}}\right) \cdot\left(t-t_{0}\right)^{2}},
\end{aligned}
$$

where $t_{0}$ and $v_{\mathrm{e}, \mathrm{ref}}$ are the zero-Doppler time and the effective velocity for the reference point, respectively. $\mathbf{p}_{\text {sat }}$ is the satellite position vector in Earth-Centered Earth-Fixed (ECEF) coordinates and the superscripts $\mathrm{Tx}$ and $\mathrm{Rx}$ indicate the different positions at transmission and reception, respectively, i.e., the bistatic character of the survey is accounted for. The target at the center of the beam is indicated by the vector $\mathbf{p}_{\mathrm{bc}}$, where the dependence with time indicates that the reference target might change in time, as for example occurs in the SL mode [9].

Two options are described in the following in order to correct for $\delta r_{\text {hyp }}$ : one perfectly matched to the point in the middle of the beam, which performs the correction in the azimuth-frequency domain; and a second one that approximates the compensation optimally to a line at mid-range by performing the correction in the azimuth-time domain. Their residual errors are analyzed quantitatively, thus allowing the choice of the most convenient approach for the final processing flow. The first, less attractive, possibility is to correct $\delta r_{\text {hyp }}$ both in terms of envelope and phase in the azimuth-frequency domain by using the following phase filter

$H_{\mathrm{OCO} 1}^{\text {freq }}\left(f_{\mathrm{a}}, f_{\mathrm{r}} ; r_{\text {ref }}\right)=\exp \left[\mathrm{j} \cdot \frac{4 \pi}{\mathrm{c}} \cdot\left(f_{0}+f_{\mathrm{r}}\right) \cdot \tilde{\delta r_{\text {hyp }}}\left(f_{\mathrm{a}}, f_{\mathrm{r}} ; r_{\text {ref }}\right)\right]$,

where $r_{\text {ref }}$ is the reference range, $f_{0}$ is the central frequency, the subscript OCO1 refers to the first-order orbit compensation and the superscript freq refers to the correction in the azimuth-frequency domain. $\tilde{\delta r_{\text {hyp }}}$ is now the error given in (6) mapped into the 2D frequency domain by computing the azimuth time of stationary phase numerically.

The correction in the Doppler domain using (7) assumes azimuth invariance. However, the spaceborne SAR geometry is azimuth-variant due to the curved orbit and Earth's rotation. Indeed, although the term $\delta r_{\text {hyp }}$ varies mildly with the azimuth time, the effective velocity inside (6) shows a stronger variation. The azimuth-variant geometry can be evaluated by computing the effective velocity for a target separated only in the along-track dimension and then computing the mis-match during azimuth compression. Analytically, this is given by

$$
\begin{aligned}
\varphi_{\text {error }}^{\mathrm{var}}\left(f_{\mathrm{a}} ; r_{0}\right)= & -\frac{4 \pi}{\lambda} \cdot r_{0} \cdot\left(\sqrt{1-\left(\frac{\lambda f_{\mathrm{a}}}{2 v_{\mathrm{e}}}\right)^{2}}\right. \\
& \left.-\sqrt{1-\left(\frac{\lambda f_{\mathrm{a}}}{2 v_{\mathrm{e}, \text { ref }}}\right)^{2}}\right)
\end{aligned}
$$

where $v_{\mathrm{e}}$ is the effective velocity of the target under consideration. The phase error in (8) can result in defocusing 


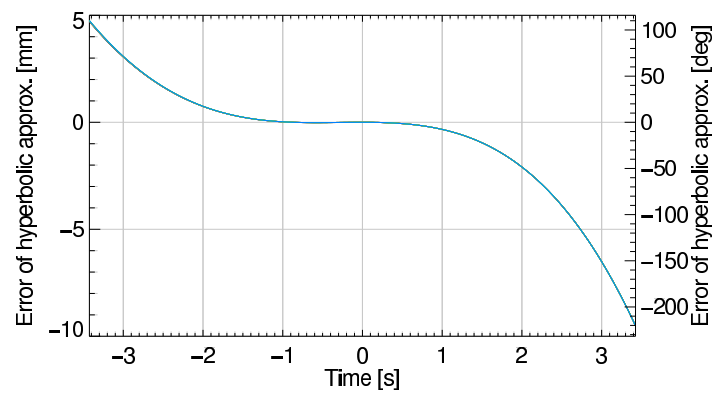

Fig. 2. Error of the hyperbolic approximation using an effective velocity. The duration corresponds to the TerraSAR-X staring spotlight (ST) data take, whose main parameters are shown in Table II.

depending on the azimuth distance to the reference point and the azimuth processed bandwidth. A numerical simulation using a TSX-like orbit was performed in order to evaluate (8) for different incidence angles and latitudes. The selected Keplerian orbit parameters are shown in Table III. Fig. 3 shows the quadratic phase error (QPE) at the edge of the azimuth processed bandwidth, which in this case corresponds to $15 \mathrm{~cm}$ azimuth resolution without weighting. The analyzed target is located $2 \mathrm{~km}$ away from the reference target in the azimuth dimension, but at the same slant-range. Both points are located at $0 \mathrm{~m}$ altitude over the WGS-84 ellipsoid. Note that the plot analyzes the azimuth variance assuming the residual error has already been corrected in the azimuth-frequency domain using (7). Note also that the given values vary linearly with the distance to the scene center, quadratically with the azimuth processed bandwidth, and linearly with the wavelength, i.e., C-band has a larger error than X-band for the same processed bandwidth since it requires a larger integration time. The plot corresponds to an ascending orbit configuration and rightlooking geometry, but similar plots are obtained for other configurations or orbits. In the configuration of the ST mode, the resulting values will degrade the azimuth resolution and introduce interferometric phase errors. As a final comment, note that due to the spotlight acquisition geometry, it does not help to process the raw data in azimuth blocks to accommodate the azimuth variance.

In order to efficiently handle the azimuth variance, the second possibility is to perform the correction with a phase multiplication in the azimuth-time domain using

$$
H_{\mathrm{OCO} 1}\left(t, f_{\mathrm{r}} ; r_{\mathrm{ref}}\right)=\exp \left[\mathrm{j} \cdot \frac{4 \pi}{\mathrm{c}} \cdot\left(f_{0}+f_{\mathrm{r}}\right) \cdot \delta r_{\mathrm{hyp}}\left(t ; r_{\mathrm{ref}}\right)\right] \text {, }
$$

which, again, shifts the signal both in terms of envelope and phase. Effectively, eq. (9) can accommodate the azimuth variance besides correcting the non-hyperbolic term due to the small beamwidth of the antenna. This correction makes the beam-center approximation [25], i.e., the correction is only valid for the target in the middle of the beam, but given the small beamwidth of current spaceborne sensors, e.g., $0.33^{\circ}$ for TSX, the introduced error is very small. With the correction given by (9), a pure hyperbolic phase history is forced, so that a conventional frequency domain kernel assuming a hyperbolic phase history can still be used to process the data without

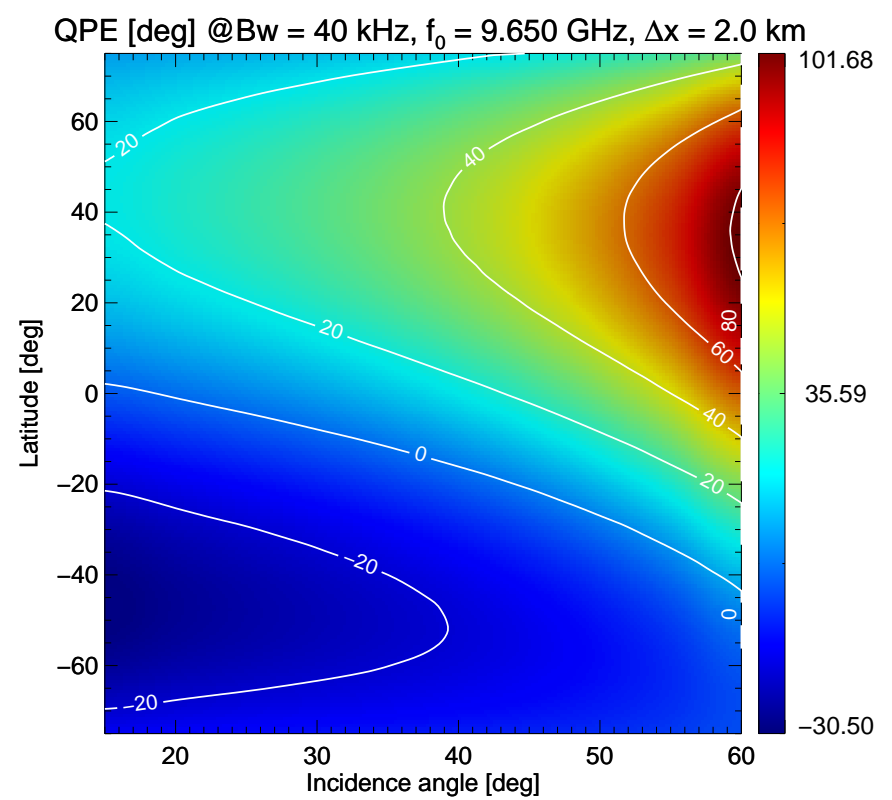

Fig. 3. Quadratic phase error (QPE) in degrees at the edge of the azimuth processed bandwidth due to the azimuth variance of the imaging geometry as a function of the incident angle and the latitude. A Keplerian TerraSAR-Xlike orbit was simulated (see Table III). The azimuth processed bandwidth is $40 \mathrm{kHz}$ and the target is located $\Delta x=2 \mathrm{~km}$ away from scene center only in the azimuth dimension. The orbit is in ascending configuration and a rightlooking geometry was assumed. This plot evaluates (8) assuming the orbit curvature was corrected using (7), which neglects the azimuth variance of the geometry.

modifications. Note that this correction is accurate in widebandwidth terms, but only for the reference target. Therefore, for other ranges or azimuth positions the correction becomes less accurate the larger the scene size.

Since the correction given by (9) is only valid for mid range, a range-dependent (second-order) OCO might be necessary, as it is usually the case with airborne SAR systems. Due to the small magnitude of the residual errors, a phase correction suffices, which is given by [17], [19]

$$
H_{\mathrm{OCO} 2}(t, r)=\exp \left[\mathrm{j} \cdot \frac{4 \pi}{\lambda} \cdot\left(\delta r_{\text {hyp }}(t ; r)-\delta r_{\text {hyp }}\left(t ; r_{\text {ref }}\right)\right)\right] \text {. }
$$

For large errors, a range interpolation might be additionally needed in order to correct the range-dependent shift. In any case, note that due to the large separation between the sensor and the scene and the small swath width, the range-dependency can be neglected in most cases (the residual phase errors at the edge of the bandwidth are smaller than $1^{\circ}$ for the TSX ST data take shown in Section V). The accuracy of the orbit compensation depends mainly on the accuracy of the orbit product, which for the TSX science orbit is about $3 \mathrm{~cm}(1 \sigma)$ in periods of low solar activity [26]. Since only the relative error given by (6) is of interest for the compensation, such an accuracy suffices.

Similar as in Fig. 3, the performance of the OCO approach can be evaluated numerically. In this case though, the residual 


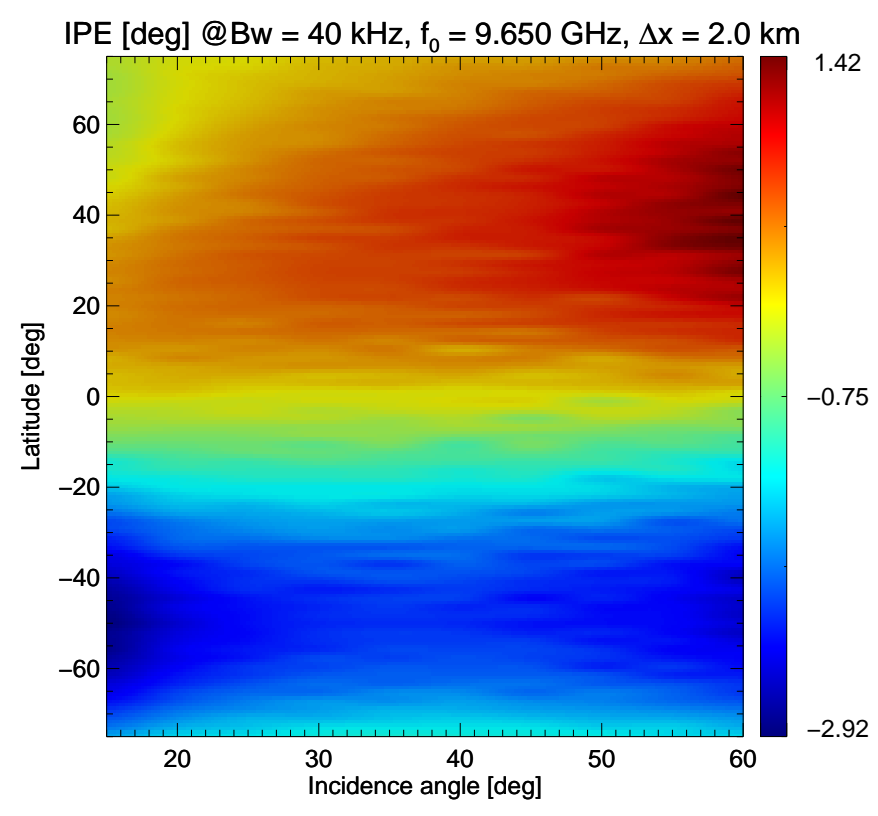

Fig. 4. Interferometric phase error (IPE) after applying the proposed orbit compensation for a target located $2 \mathrm{~km}$ away from the scene center in the azimuth direction and assuming an azimuth processed bandwidth of $40 \mathrm{kHz}$. A Keplerian TerraSAR-X-like orbit was simulated (see Table III). The orbit is in ascending configuration and a right-looking geometry was assumed. No weighting for sidelobe suppression was used.

error is not quadratic, and the interferometric phase error (IPE) is shown instead, which is the integration of the error along the azimuth frequency. The error for the OCO approach is given in Fig. 4, where no weighting was assumed. Note that the maximum IPE is smaller than $3^{\circ}$, while it reduces to less than $2^{\circ}$ with the usual weighting of TSX, i.e., a raised cosine window with $\alpha_{w}=0.6$. The OCO approach leads to very good results for the TSX case, but needs to be evaluated for each scenario, being the one shown in Fig. 4 already a quite demanding one.

Finally, note that the suggested orbit compensation scheme is the simplest to implement compared to existing advanced motion compensation approaches for airborne SAR [27]-[31], and despite the good performance of the proposed approach, it might not be sufficient to accommodate properly the azimuth variance or higher order effects for more demanding scenarios (better resolution, larger coverage). In such cases, any of the aforementioned techniques can be used to improve the performance, some of which use a block-wise processing approach, which in any case might be required due to the topography within the scene, as commented later in Section III-C.

\section{Atmospheric Effects}

The troposphere introduces an undesired delay in the electromagnetic signal in the order of $2-4$ meters (one-way). The zenith path delay through the troposphere as a function of the target height has been modeled in the literature in different ways, e.g., using a quadratic [32] or an exponential [20], [33] function. The operational TSX processor uses the latter model, which is given by

$$
\begin{aligned}
\Delta R_{\text {tropo }}\left(t ; r_{\text {ref }}\right) & =\frac{Z \cdot \exp \left[-h\left(r_{\text {ref }}\right) / H\right]}{\cos \theta\left(r_{\text {ref }}\right) \cdot \cos \alpha_{i}\left(t ; r_{\text {ref }}\right)} \\
& =\frac{\Delta R_{\text {tropo }}^{\text {ref }}\left(r_{\text {ref }}\right)}{\cos \alpha_{i}\left(t ; r_{\text {ref }}\right)}
\end{aligned}
$$

where $Z$ is a constant zenith path delay in meters, $H$ is a reference height, $h$ is the altitude of the target, $\theta$ is the look incidence angle, and $\alpha_{i}$, which has been introduced here in addition, is the incident azimuth angle during the formation of the synthetic aperture. The rationale for the additional term, $1 / \cos \alpha_{i}$, in the mapping function is sketched in Fig. 5, where the troposheric delay at zero Doppler is projected for each time instant during the formation of the synthetic aperture. The presented model is physically valid assuming a troposphere that is cylindrical symmetric around the reference point and time invariant during the synthetic aperture, which shall be the case at decimeter scales and suffices to achieve the nominal azimuth resolution, as commented later. Note that for increasing integration times the difference between $\alpha$ and $\alpha_{i}$ also increases (cf. Fig. 5). Furthermore, for large squints close to grazing angles, more sophisticated mapping functions shall be used [33], [34]. The model is validated experimentally in Section $\mathrm{V}$ for a squint angle variation of $\pm 2.2^{\circ}$ corresponding to the ST mode, but further investigations would be required in order to validate the model for larger integration angles. Fig. 6 plots (11) after subtracting a constant offset, as a function of the azimuth angle $\alpha_{i}$ assuming the following values: $H=6$ $\mathrm{km}, Z=2.6 \mathrm{~m}, h=629 \mathrm{~m}$ and $\theta=35^{\circ}$. As it can be induced from Fig. 6, for large integration times the dependence with $\alpha_{i}$ is not negligible, hence introducing defocusing and phase errors if not considered. Furthermore, the delay in absolute terms, which in the TSX case shifts the image about 6 pixels in the slant-range dimension for the maximum sampling frequency, implies a mis-match of the azimuth compression filter, introducing additional defocusing and phase errors.

Fig. 7 shows this effect over the same CR as shown in Fig. 1, where the corrections mentioned in the previous sections have been already applied. For the configuration of this data take, the troposphere introduces a quadratic error of about $50^{\circ}$ at the edge of the processed bandwidth, and the further $50^{\circ}$ occur due to the azimuth filter mis-match, resulting in the total $100^{\circ}$ that can be observed in Fig. 7, which degrades the azimuth resolution by about $10 \%$. The correction of the troposphere using (11) can be applied together with the first order OCO, since a bulk correction for the middle of the scene suffices. Therefore, (9) becomes

$$
\begin{aligned}
H_{\mathrm{OCO} 1}\left(t, f_{\mathrm{r}} ; r_{\text {ref }}\right)= & \exp \left[\mathrm { j } \cdot \frac { 4 \pi } { \mathrm { c } } \cdot ( f _ { 0 } + f _ { \mathrm { r } } ) \cdot \left(\delta r_{\text {hyp }}\left(t ; r_{\text {ref }}\right)\right.\right. \\
& \left.\left.+\Delta R_{\text {tropo }}\left(t ; r_{\text {ref }}\right)\right)\right]
\end{aligned}
$$

Note that the performance of the tropospheric correction will depend on the accuracy of the assumed model. In any case, the troposheric component is space variant, with gradients of about $1 \mathrm{~cm} / \mathrm{km}$ [35]. It is not the purpose of the suggested correction to achieve such an accuracy in absolute terms, but 


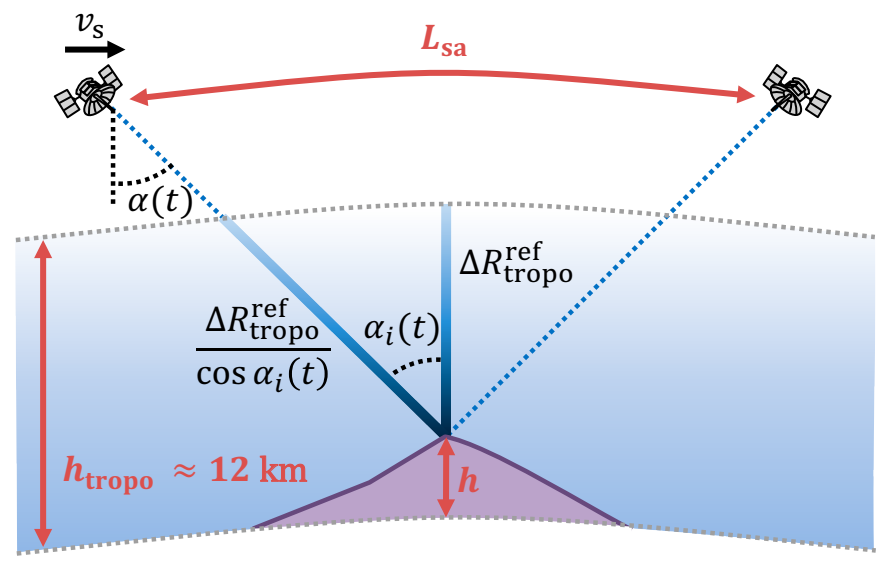

Fig. 5. Tropospheric delay variation within the synthetic aperture, $L_{\mathrm{sa}}$, as a function of the incident azimuth angle, $\alpha_{i}(t) . \Delta R_{\mathrm{tropo}}^{\mathrm{ref}}$ is the tropospheric delay at zero-Doppler, $h_{\text {tropo }}$ is the height of the troposphere, $h$ the height of the target, and $\alpha$ is the squint angle defined in the slant-range plane. The thick solid lines represent the delay introduced in the signal when traveling through the troposphere. Note that despite the exaggerated Earth curvature shown in the picture, the mapping factor $1 / \cos \alpha_{i}$ can be applied by assuming a flat geometry, even for large squint angles, as the troposphere thickness is very small compared to the Earth radius.

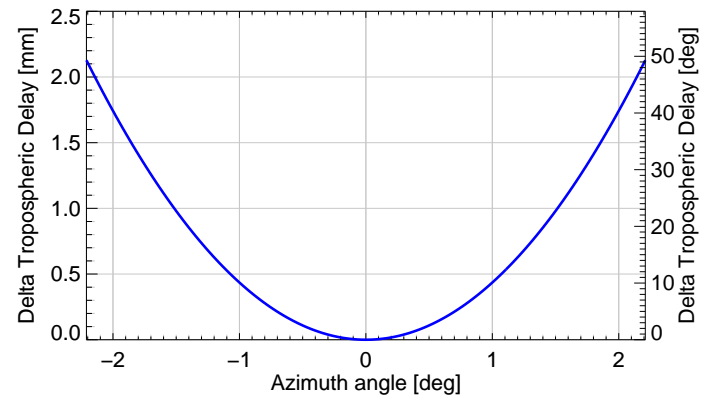

Fig. 6. Delta tropospheric delay, i.e., after subtracting a constant offset, as a function of the azimuth angle using (11). The angle variation corresponds to the TerraSAR-X staring spotlight mode. If not considered, the phase error will turn into azimuth defocusing and phase errors.

rather to reduce the relative disturbance, i.e., the curvature, to a level where it does not affect the focusing quality. In this sense, the correction should be accurate at sub-pixel level in the range dimension, which is sufficient in order to obtain a proper curvature correction of the disturbance. On the other hand, strong topography variations within the imaged swath cannot be adapted with this simple correction. Since the topography also plays an important role within the focusing kernel itself, as later discussed in Section III-C, the tropospheric correction term due to topography variations can be compensated as well.

Concerning the ionosphere, the total delay is roughly $2 \mathrm{~cm}$ at X-band [20], and hence the dependence with $\alpha_{i}$ can be neglected. On the other hand, the effect on wide-band systems is an issue not addressed in this paper due to its negligible effect in the TSX case.

\section{FURTHER CONSIDERATIONS}

The three aspects mentioned in the previous sections are the main ones in order to ensure a proper focusing, but not the

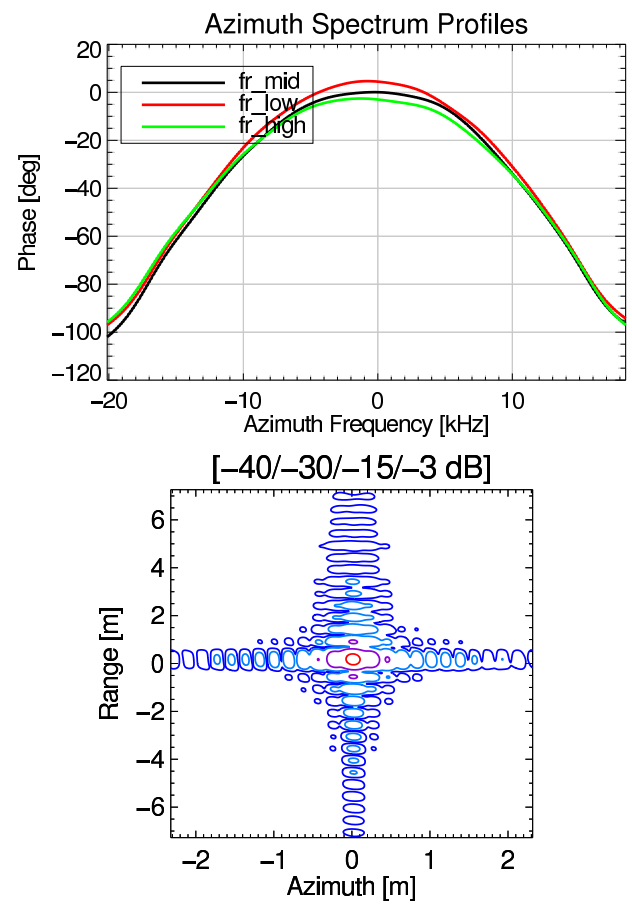

Fig. 7. Effect of the troposphere in the TerraSAR-X staring spotlight mode. (top) Phase error in the Doppler domain measured over a CR for different range frequencies and (bottom) the corresponding contour plot of the oversampled IRF.

only ones. This section addresses three further aspects, which are: special considerations when processing using sub-aperture approaches to avoid azimuth aliasing; the consideration of the array pattern to avoid resolution loss in the focused image; and the topography dependence of the focusing kernel.

\section{A. Sub-Aperture Processing}

The division of the raw data in sub-apertures is a wellknown approach to accommodate the Doppler variation introduced in the signal by the antenna steering, which exploits the fact that the PRF is larger than the beam bandwidth [4], [22], [23]. The recombination is performed in the time domain after the range-variant processing has been performed. When using the azimuth scaling approach during the azimuth processing, a small margin is considered due to the stretching of the azimuth signal. Finally, the computed size is zero-padded to the next power of two for efficiency purposes. Nevertheless, a further fact needs to be considered: the RCMC filter stretches also the azimuth signal in time domain for range frequencies other than $f_{0}$. This happens especially for sub-apertures with large Doppler centroids. Hence, when going back to azimuth-time domain, where the sub-apertures are recombined, time-aliasing might appear, resulting in a considerable degradation of the focused image. The amount of this stretching can be computed using the RCMC filter, resulting in an additional extension that can be even larger than the effective sub-aperture size itself. Analytically, it suffices to take the RCMC filter used, e.g., by chirp scaling [36], so that the stretching can be computed for 
each sub-aperture as

$$
\begin{aligned}
\Delta t_{\mathrm{rcmc}}\left(f_{\mathrm{DC}, \mathrm{i}}\right) & =\left.\frac{1}{2 \pi} \cdot \frac{\partial \phi_{\mathrm{rcmc}}\left(f_{\mathrm{a}}, f_{\mathrm{r}}\right)}{\partial f_{\mathrm{a}}}\right|_{f_{\mathrm{a}}=f_{\mathrm{DC}, \mathrm{i}}} \\
& =\frac{2 r_{\mathrm{ref}}}{\mathrm{c}} \cdot \frac{f_{\mathrm{DC}, \mathrm{i}} \cdot\left(\frac{\lambda}{2 v_{\mathrm{e}}}\right)^{2}}{\left(1-\left(\frac{\lambda f_{\mathrm{DC}, \mathrm{i}}}{2 v_{\mathrm{e}}}\right)^{2}\right)^{3 / 2}} \cdot B_{\mathrm{rg}},
\end{aligned}
$$

where $\phi_{\mathrm{rcm}}$ is the phase function of the RCMC filter, and in practice $f_{\mathrm{a}}$ has been substituted by the Doppler centroid of the given sub-aperture, $f_{\mathrm{DC}, \mathrm{i}}$, and the range frequency $f_{\mathrm{r}}$ by the range bandwidth, $B_{\mathrm{rg}}$, in order to compute the required time extension. After converting the extension into samples, the sub-aperture shall be zero-padded with half of this amount on each side.

A further aspect that needs to be considered is the Doppler centroid variation due to the wavelength dependence. The well-known Doppler formula is given by

$$
f_{\mathrm{DC}}=\frac{2 v_{\mathrm{s}}}{\lambda} \cdot \sin \alpha,
$$

where $v_{\mathrm{s}}$ is the satellite velocity and $\alpha$ is the squint angle. Therefore, for a given squint angle, the Doppler centroid changes as a function of the wavelength, i.e., within the range bandwidth, a fact that needs to be considered in order to avoid that parts of the spectrum cross the adjacent PRF band. A solution was proposed in [37] based on the azimuth spectral extension, which consists in replicating the two-dimensional spectrum in azimuth and filtering out the undesired parts. By doing so the azimuth sampling is increased according to the spectral extension. In the case of the TSX satellite and due to the small relative bandwidth, it suffices to increase the PRF in order to accommodate the Doppler-centroid variation, since the skew of the spectrum at the maximum squint angle is about $B_{\text {skew }}=600 \mathrm{~Hz}$. Note further, that the PRF must be increased anyway to reduce the impact of the azimuth ambiguities in the TSX case [9], [11]. The skew of the spectrum imposes an additional condition when computing the size of the subaperture [4], which now is given by

$$
T_{\text {sub }}=\frac{\operatorname{PRF}-B_{\text {az }}-B_{\text {skew }}}{\left|K_{\text {rot }}\right|}[s],
$$

where $B_{\mathrm{az}}$ is the azimuth processed bandwidth and $K_{\mathrm{rot}}$ is the Doppler centroid variation introduced by the steering of the antenna. If the processing is performed in the Fourier domain, the proper approach is to perform the spectral extension as proposed in [37].

\section{B. Array Pattern}

In electronically-steered antennas, a significant amplitude modulation is introduced by the single element antenna pattern when the steering angles become comparable to the single element pattern beamwidth. Furthermore, other aspects like, e.g., the feed network or the coupling between antenna elements, can influence the final signal amplitude. Fig. 8 shows the computed normalized modulation using the TSX antenna patterns for a given ST data take. This curve has been

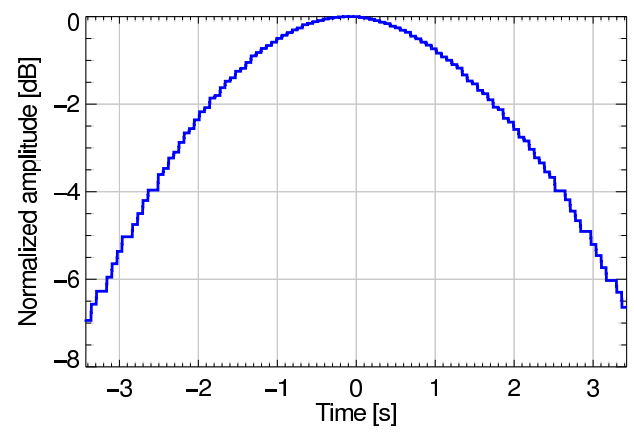

Fig. 8. Amplitude modulation introduced by the antenna array when steering the TerraSAR-X antenna within $\pm 2.2^{\circ}$. The staircase behavior occurs due to the quantization of the phase shifts used for the steering of the pattern.

computed by integrating for each echo the transmit-receive antenna pattern within the main lobe, i.e.,

$$
A[n]=\left(\int_{\Omega_{3 \mathrm{~dB}}} G_{\mathrm{Tx}}(\alpha ; n] \cdot G_{\mathrm{Rx}}(\alpha ; n] \cdot \mathrm{d} \alpha\right)^{\frac{1}{2}},
$$

where $G(\alpha ; n]$ is the one-way azimuth antenna pattern as a function of the azimuth angle $\alpha$ and the discrete echo line number $n$. After normalization, the inverse of this curve can be applied directly at raw data level to correct for the undesired modulation, similar as the recently proposed approach for the scalloping correction of TOPS data [38]. Note that, in any case, a scaling of the noise at scene edges is unavoidable. Note also that the inter-element distance of the azimuth antenna tiles (0.4 m for TSX [39]) plays an important role, since, in combination with the element antenna pattern, it results in the so-called grating lobes. Precisely at the maximum steering angles of $\pm 2.2^{\circ}$ the strongest grating lobe has exactly the same gain as the main lobe. Therefore, the element antenna pattern and the grating lobes have an important impact in terms of azimuth ambiguities and must be precisely considered for the commanding of the ST mode in the TSX case [9], [11]. On the other hand, the influence of noise scaling can be partially mitigated at detected image level by proper noise subtraction as proposed in [40], but note that this approach has not been applied to the TerraSAR-X images shown in Section V.

\section{Topography Dependence}

For a given zero-Doppler slant-range distance $r_{0}$, the range history in a spaceborne scenario depends on the topography due to the curved orbit [5], [41], [42]. Similar as with the hyperbolic range history approximation, this effect becomes more noticeable for larger integration times. This means that, if the processing is performed with an azimuth-invariant reference height, as is usually assumed within current spaceborne imaging algorithms, azimuth defocusing might occur. Fig. 9 shows the height error that results in a phase error of $\pi / 2$ at the edge of the processed bandwidth as a function of the azimuth resolution for the TSX-like orbit of Table III. This corresponds to a resolution loss of approximately $10 \%$ without weighting function, since the phase error is mainly quadratic with frequency. In the ST mode the topography should be accommodated within $60 \mathrm{~m}$ to avoid defocusing greater than 


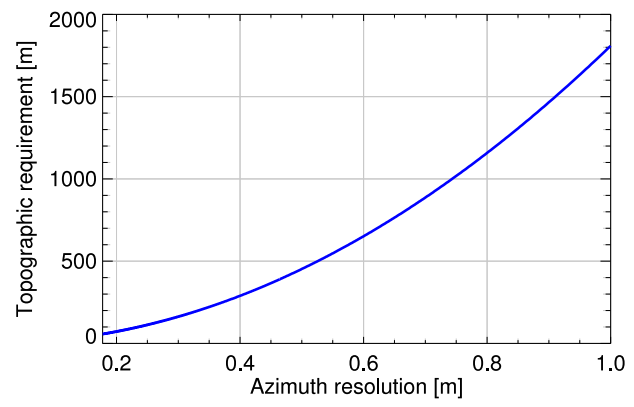

Fig. 9. Maximum allowed topographic height error as a function of the azimuth resolution by allowing a maximum phase error of $\pi / 2$ at the edge of the azimuth processed bandwidth. A TSX-like orbit has been simulated (see Table III)

$10 \%$. For a more detailed analysis concerning the topography dependency in SAR focusing please refer to [41].

Due to the space variance of the effect, it is not trivial to include the compensation within the kernel of a Fourierbased processor. Therefore, one straightforward approach is to perform a block-wise post-focusing using an external DEM as suggested in [42], an approach based on existing topographydependent motion compensation approaches for airborne SAR systems [43], [44]. Please note that for the results shown in Section V no post-focusing was performed, and instead the reference height was matched to that of the reference points to be analyzed.

At this point it is interesting to note that the troposphere correction also depends on the height of the target [see (11)]. Hence, the block-wise approach could also handle a residual tropospheric correction. When no external information concerning the topography is available, then autofocus approaches can be used instead to improve the focusing of the image.

\section{Proposed Processing Flow}

Fig. 10 shows the suggested processing chain for the focusing of very high resolution spotlight SAR data, which includes the corrections mentioned in the previous sections. The block diagram reflects the generality of these corrections and hence it is not specified for any particular focusing kernel. Without loss of generality, the consideration of the higher signal bandwidth w.r.t. the PRF is presented either by using sub-apertures or with the deramping solution expounded in [24], where in the latter case no assembly of sub-apertures is needed, but the kernel must account for the changing of the azimuth sampling after the deramping operation.

The blocks highlighted in gray are the new ones proposed in this paper. First, the array pattern correction is performed at raw data level if required, i.e., in case an electronicallysteered array is used. After handling the large azimuth signal bandwidth either with sub-apertures (in which case each subaperture needs to be zero-padded considering the stretching due to the RCMC filter, see Section III-A) or with the deramping approach presented in [24], a 2D fast Fourier transform (FFT) together with, whenever required, the azimuth spectral extension, follows. The stop-and-go correction defined in (5) can then be applied. After an inverse azimuth FFT, the first order OCO and the tropospheric correction are applied together through the azimuth-dependent phase function given by (12) to correct both the phase and the envelope of the signal for mid-range. An inverse range FFT brings the signal back to the range- and azimuth-time domain, where any Fourier-based processing kernel can now be used in order to perform the range-variant processing. This includes monochromatic kernels, like chirp scaling (CS) approaches [18], [36], the range-Doppler (RD) algorithm with secondary range compression (SRC) in the 2D frequency domain [16], [45], or algorithms based on the chirp-Z transform (CZT) [24], [46], as well as polychromatic ones, which basically leads to the $\omega-\mathrm{k}$ algorithm [47] and its extensions to handle the spaceborne geometry, especially in terms of the separation of the azimuth compression step from the Stolt mapping in order to ease the implementation of the Stolt interpolation itself [48] or to facilitate the motion compensation in airborne systems (extended $\omega-\mathrm{k}$, EOK [29]), or by computing numerically the Stolt kernel in order to accommodate more precisely the orbit curvature and the variation of the effective velocity with range, an approach named singular value decomposition (SVD)-Stolt [15]. The range-variant processing shall include a residual correction of the RCM in the range-Doppler domain via interpolation due to the range dependence of the effective velocity, a step implicitly included in the range-Doppler algorithm. The selection of the range-variant processing kernel will depend on the sensor capabilities and image quality requirements. For the most demanding scenarios it is suggested to use the OCO together with a modified SVD-Stolt [15] where the azimuth compression is removed from the Stolt mapping as in EOK [29], [48]. This approach can account for the residual SRC due to the curved orbit for targets located at ranges other than $r_{\text {ref }}$, since the OCO is only accurate for this range, while still being able to handle the azimuth-variant geometry.

After the range-variant processing, the second order orbit compensation can take place in terms of a residual phase correction as given by (10) in order to accommodate the range variance. Again, this correction can include a delta tropospheric delay correction. The azimuth processing can take place afterwards, which can be solved with a SPECAN approach in case of using sub-apertures [18], [23] or with a conventional matched filtering, and in both cases considering the range-dependence of the effective velocity.

As a last comment, the higher the resolution, the larger the sensitivity to the topography, as shown in Fig. 9. For very high resolution spaceborne SAR imaging, this will be the limiting factor, which, as already commented, requires a space-variant correction. Such a correction could deal with other residual errors not considered by the focusing kernel, e.g., azimuth variance not corrected by the $\mathrm{OCO}$, residual SRC, residual RCM, etc. However, the better the performance of the Fourierbased kernel, the simpler the post-processing will be.

\section{EXPERIMENTAL RESULTS}

A simulation with point targets was performed using the parameters shown in Table IV and a TSX-like orbit (see Table III). Note that the parameters have been chosen based on 


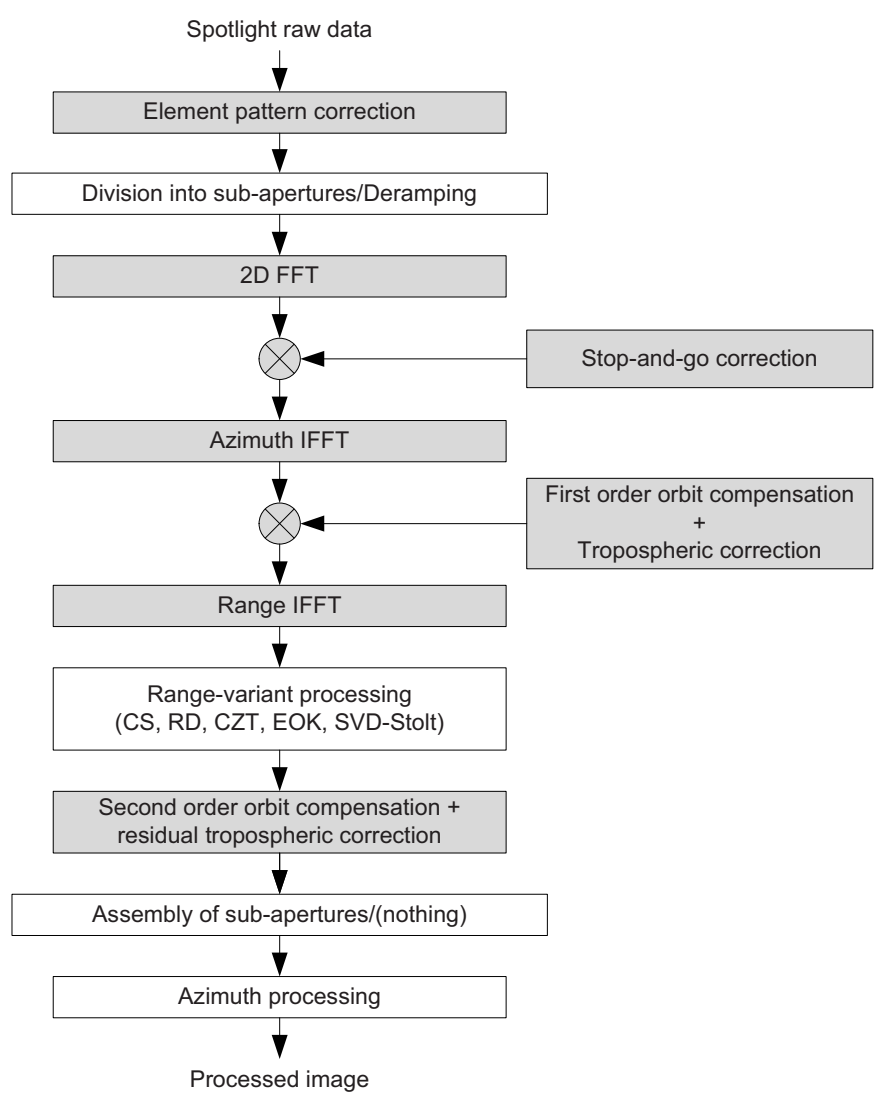

Fig. 10. Proposed processing chain (new steps in gray) to process high resolution spotlight data. CS stands for chirp scaling, RD for range-Doppler, CZT for chirp-Z transform, EOK for extended $\omega-\mathrm{k}$, and SVD for singular value decomposition.

TABLE IV

SIMULATION PARAMETERS

\begin{tabular}{lc}
\hline Central frequency & $9.65 \mathrm{GHz}$ \\
Total azimuth processed bandwidth & $33 \mathrm{kHz}$ \\
Azimuth resolution & $19 \mathrm{~cm}$ \\
Chirp bandwidth & $1.2 \mathrm{GHz}$ \\
Ground range scene size & $5 \mathrm{~km}$ \\
Azimuth scene size & $5 \mathrm{~km}$ \\
Incidence angle & $55^{\circ}$ \\
Central point (longitude/latitude) & $11.264^{\circ}, 48.088^{\circ}$ \\
\hline
\end{tabular}

a possible configuration for a next generation X-band satellite [49]. The motion of the satellite was simulated between and during transmission and reception, while the troposphere was not included. Due to the large relative bandwidth, monochromatic algorithms do not perform properly, so the EOK approach was selected, further including a residual RCMC in the form of an interpolation in the range-Doppler domain after the Stolt mapping to adapt for the variation of the effective velocity. The second order OCO is not really needed in this case and hence was not applied. Fig. 11 shows the IRFs of the nine point targets, which are located at the edges and the center of the $5 \mathrm{~km} \times 5 \mathrm{~km}$ scene. The measured resolutions are within $0.5 \%$ accuracy while the IPEs are smaller than $1^{\circ}$.

Real data acquired by TSX in the staring spotlight mode have been further used to validate the proposed methodology,
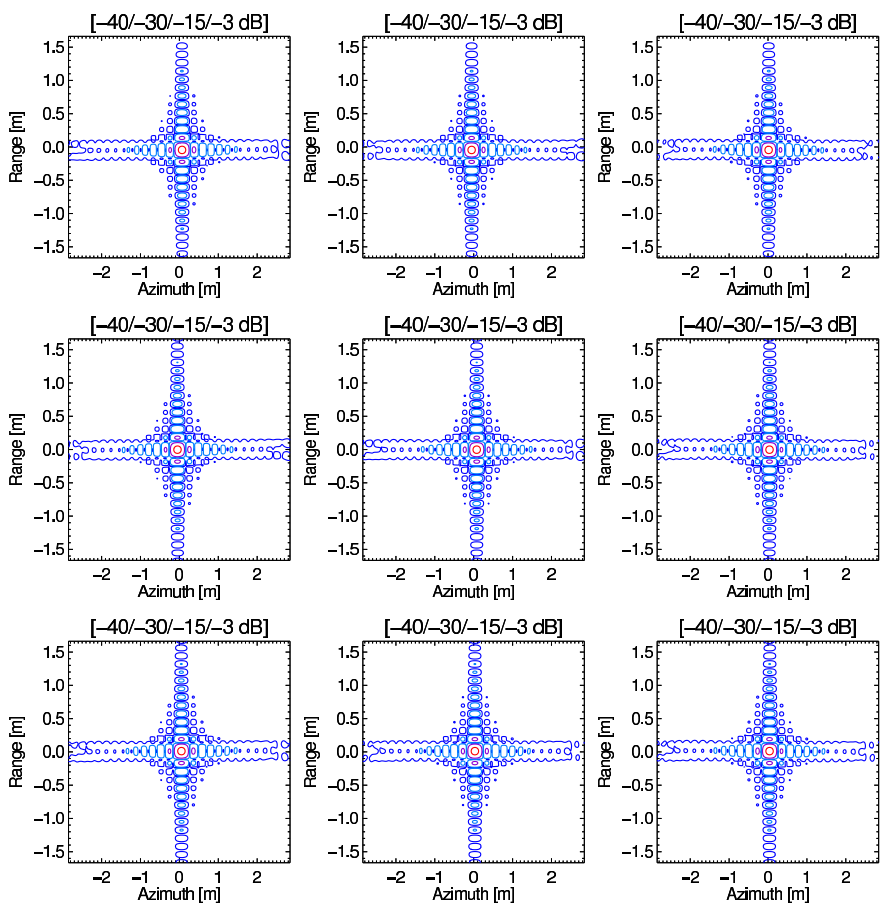

Fig. 11. Contour plots of the interpolated IRFs by using the proposed processing chain with the extended $\omega-\mathrm{k}(\mathrm{EOK})$. The simulation parameters appear in Table IV.
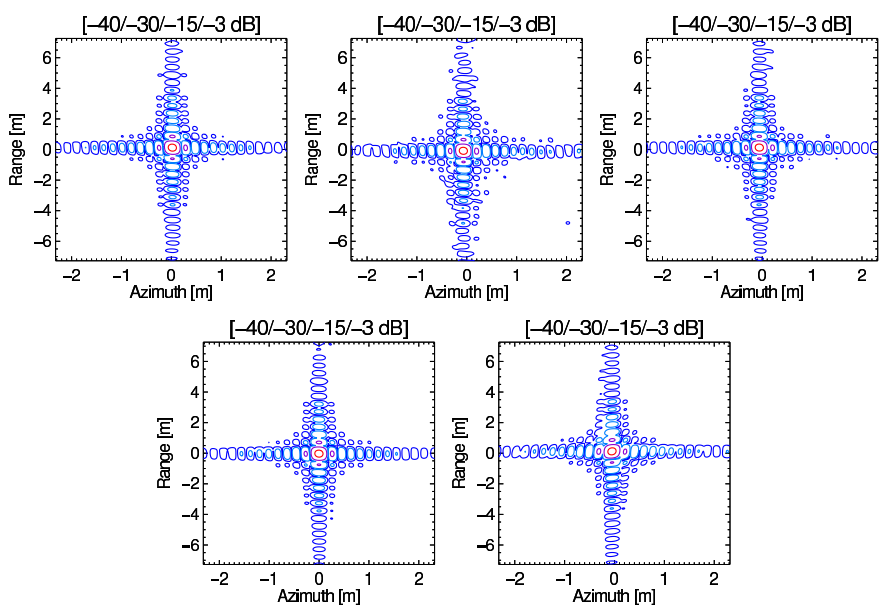

Fig. 12. Contour plots of the interpolated IRFs of five CRs of the TerraSAR$\mathrm{X}$ staring spotlight image acquired over Oberpfaffenhofen, Germany. The last $\mathrm{CR}$ on the lower row is located at the right edge of the scene in near range. No sidelobe suppression was performed, so that the obtained resolutions are $16 \mathrm{~cm}$ in azimuth and $44 \mathrm{~cm}$ in slant-range, hence matching the theoretical values. The data were processed with the proposed processing chain and the range-Doppler (RD) algorithm. 

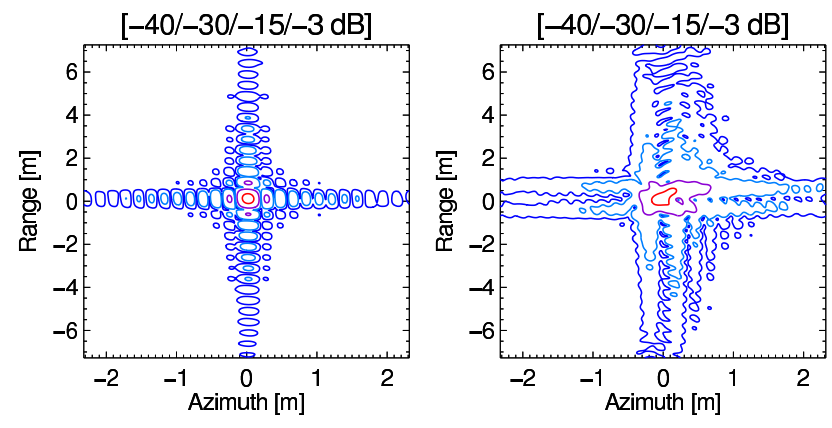

Fig. 15. Contour plots of the interpolated IRF of a CR after processing with the range-Doppler (RD) algorithm (left) using the proposed processing chain and (right) without any of the main corrections expounded in Section II.

where in this case the influence of the troposphere needs to be considered. Table II shows the main acquisition parameters, while Fig. 12 depicts the IRFs of the five CRs located in the scene acquired over Oberpfaffenhofen, Germany. During the processing all the anomalies were considered and corrected. The data were processed following the flow diagram of Fig. 10 using the RD algorithm in sub-apertures. The second order OCO was not performed and the reference point for both the first order OCO and the tropospheric correction is in the middle of the scene. Without spectral weighting, the measured values are $44.9 \mathrm{~cm}$ and $16.4 \mathrm{~cm}$ in range and azimuth, respectively, hence matching the theoretical ones with $2 \%$ accuracy. The reflectivity image is shown in Fig. 13, while Fig. 14 shows a zoom of it and compares it with the standard high-resolution spotlight (HS) mode. In the latter case, both images have been multilooked to a resolution of $1 \mathrm{~m} \times 1 \mathrm{~m}$, and the radiometric resolution improvement of the ST image is evident. Note also that there is a gain in the detectability of point targets (about $7 \mathrm{~dB}$ ) in the full resolution ST data due to the increased compression gain.

Finally, in order to remark the importance of the suggested corrections, Fig. 15 shows the interpolated IRF of one of the CRs of Fig. 12 with and without the main corrections expounded in Section II, namely, the "fast-time" stop-and-go effect, the tropospheric correction, and the orbit compensation. Without these corrections the IRF is severely degraded, especially in terms of resolution, with a loss of more than $50 \%$ in both range and azimuth dimensions.

\section{CONCLUSION}

Spaceborne SAR systems are unique in providing highresolution, weather independent images of the Earth surface on a global scale. In order to increase the reliability and robustness of the information retrieval in SAR images, more stringent requirements for each specific application are defined by the users in terms of observables (e.g., time series), space diversity (e.g., interferometry, tomography), multi-channel acquisition (e.g., polarimetry, multi-frequency) and last but not least improved geometric resolution. State-of-the-art satellites like TerraSAR-X, TanDEM-X, COSMO-SkyMed as well as Radarsat- 2 are providing images with a resolution in the meter regime. The next generation of SAR satellites in X-band will provide a geometric resolution in the decimeter regime.
In order to cope with the demanding requirements for improved geometric resolution, this paper has presented several critical aspects for the processing of very high resolution spaceborne data, of which the motion of the platform during the transmission/reception of the chirp signals and the troposphere are the most relevant. The correction of the former can be efficiently performed in the 2D frequency domain, while the troposphere can be corrected using a simple model for a reference target inside the scene, partly accommodating its azimuth variance. The non-hyperbolic phase history, which becomes more significant at larger integration times, has been also addressed, and a simple solution based on the motion compensation of airborne SAR systems has been proposed. This orbit compensation can also accommodate to a large extent the azimuth variance of the geometry without introducing undesired interferometric phase errors. A numerical evaluation of this approach has been presented, which confirms its validity for the processing of relatively large scenes in terms of spotlight imaging.

Further practical aspects to be considered have been also addressed, namely, the array pattern of the electronically steered antenna as well as the handling of the sub-apertures in the presence of large range bandwidths or large Dopplers. Finally, the dependence of the focusing kernel with the topography of the scene has been mentioned. Indeed, the accommodation of the topography with Fourier-based processors is challenging due to its inherent space-variant property. This issue has not been directly addressed in this paper, but ongoing work can be found in [42]. Similarly, the coupling between troposphere and topography is currently being investigated [50].

Finally, a generic processing flow has been suggested. Without entering into implementation details, the proposed focusing chain can be used with any processing kernel. The large azimuth bandwidth w.r.t. system PRF can be handled by using different strategies, be it sub-apertures or a deramping approach. The separation of the azimuth compression step allows, among other things, for a residual RCMC in the rangeDoppler domain in order to accommodate the variation of the effective velocity with range, a necessary step for very high resolution spaceborne SAR imaging.

Simulated data using point targets as well as TSX ST data have been used to validate the methodology. In the latter case, the theoretical azimuth resolution of $16 \mathrm{~cm}$ without spectral weighting, $21 \mathrm{~cm}$ with the usual TSX weighting function, has been achieved after performing all the suggested corrections.

\section{ACKNOWLEDGMENTS}

We highly acknowledge the great effort and enthusiasm of all our colleagues at DLR and EADS Astrium who contributed to the success of TerraSAR-X mission and to the demonstration of the staring spotlight mode. The TerraSAR-X data take was acquired in the frame of a project for Astrium GEOInformation Services under contract F.31350/G10004444-00. TerraSAR-X is partly funded by the German Federal Ministry for Economics and Technology and realized in a public-private partnership between German Aerospace Center (DLR) and Astrium $\mathrm{GmbH}$. 


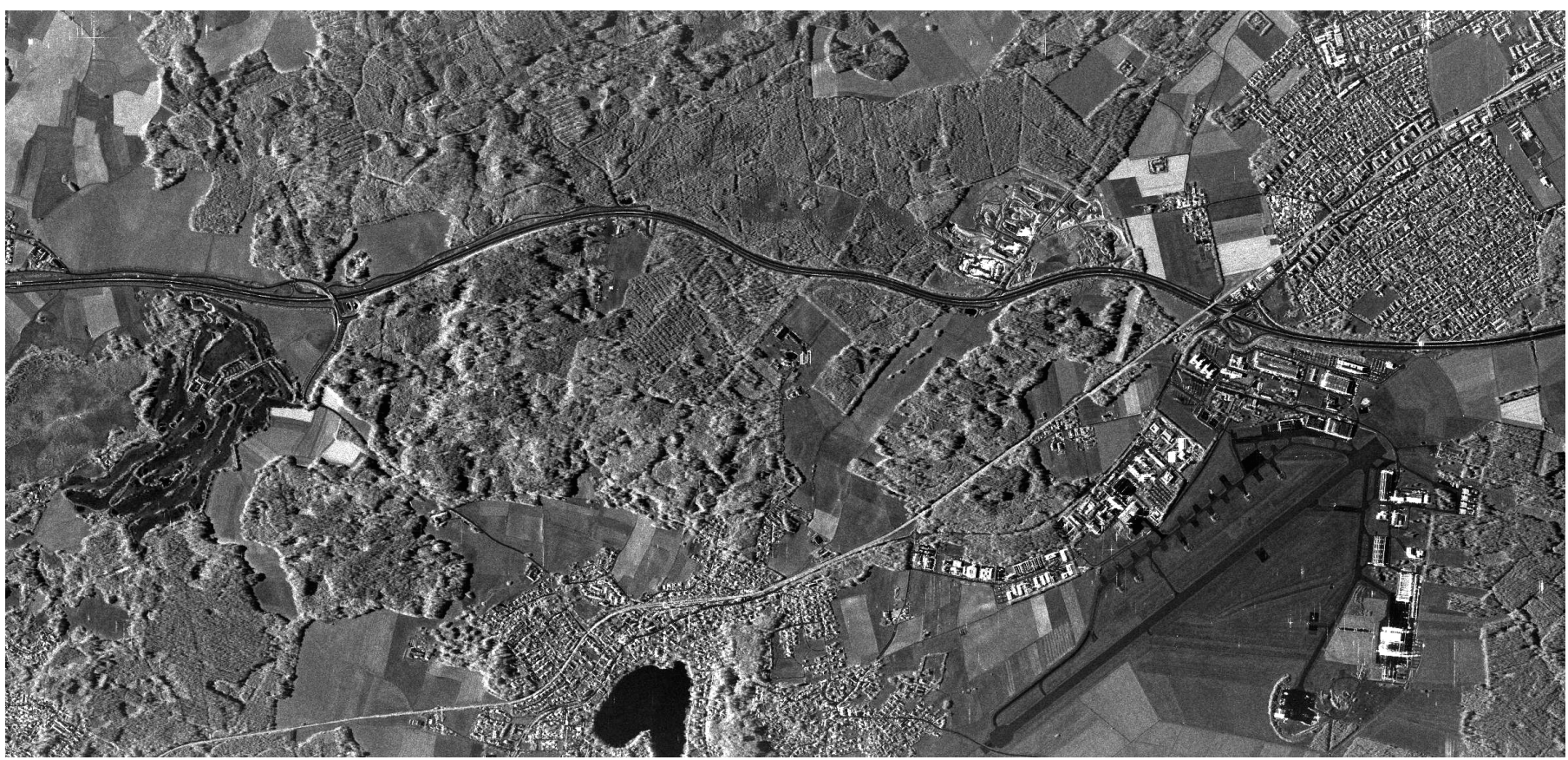

Fig. 13. Reflectivity image of the TerraSAR-X staring spotlight acquisition acquired over Oberpfaffenhofen, Germany, with $21 \mathrm{~cm}$ azimuth resolution and $58 \mathrm{~cm}$ slant-range resolution. The usual TerraSAR-X weighting was applied for sidelobe suppression.
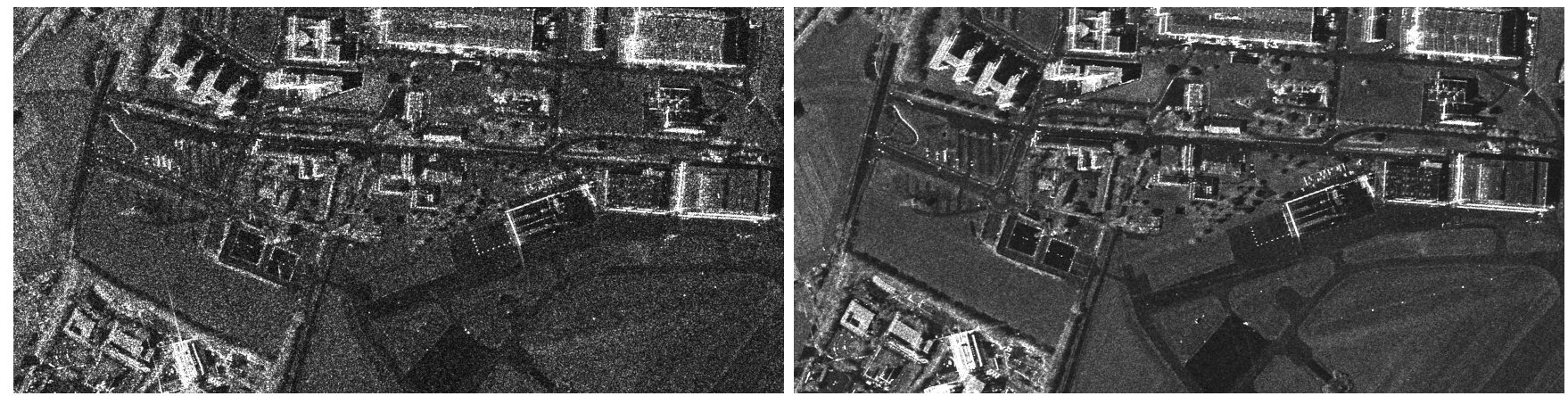

Fig. 14. Geocoded zoom of the scene shown in Fig. 13 for the (left) operational HS mode and the (right) ST mode. The resolution of the multilooked images in both cases is approximately $1 \mathrm{~m}$. Zoom size: $1000 \mathrm{~m} \times 500 \mathrm{~m}$.

The authors would also like to acknowledge the anonymous reviewers for their constructive comments and suggestions, which helped to improve the readability of the paper.

\section{REFERENCES}

[1] M. Schwerdt, B. Bräutigam, M. Bachmann, B. Döring, D. Schrank, and J. H. Gonzalez, "Final TerraSAR-X calibration results based on novel efficient methods," IEEE Trans. Geosci. Remote Sens., vol. 48, no. 2, pp. 677-689, Feb. 2010.

[2] M. Schwerdt, D. Schrank, M. Bachmann, J. H. Gonzalez, B. Döring, N. T. Ramon, and J. W. Anton, "Calibration of the TerraSAR-X and the TanDEM-X satellite for the TerraSAR-X mission," in Proc. of European Conference on Synthetic Aperture Radar (EUSAR'12), Nuremberg, Germany, 2012.

[3] A. Meta, J. Mittermayer, P. Prats, R. Scheiber, and U. Steinbrecher, "TOPS imaging with TerraSAR-X: Mode design and performance analysis," IEEE Trans. Geosci. Remote Sens., vol. 48, no. 2, pp. 759 769, Feb. 2010.

[4] P. Prats, R. Scheiber, J. Mittermayer, A. Meta, and A. Moreira, "Processing of sliding spotlight and TOPS SAR data using baseband azimuth scaling," IEEE Trans. Geosci. Remote Sens., vol. 48, no. 2, pp. 770-780, Feb. 2010.
[5] P. Prats-Iraola, R. Scheiber, L. Marotti, S. Wollstadt, and A. Reigber, "TOPS interferometry with TerraSAR-X," IEEE Trans. Geosci. Remote Sens., vol. 50, no. 8, pp. 3179-3188, 2012.

[6] M. Rodriguez-Cassola, P. Prats, D. Schulze, N. Tous-Ramon, U. Steinbrecher, L. Marotti, M. Nannini, M. Younis, P. López-Dekker, M. Zink et al., "First bistatic spaceborne SAR experiments with TanDEM-X," IEEE Geosci. Remote Sens. Lett., vol. 9, no. 1, pp. 33-37, 2012.

[7] J. Mittermayer, S. Wollstadt, P. Prats-Iraola, P. López-Dekker, G. Krieger, and A. Moreira, "Bidirectional SAR imaging mode," IEEE Trans. Geosci. Remote Sens., vol. 51, no. 1, pp. 601-614, Jan. 2013.

[8] J.-H. Kim, M. Younis, P. Prats-Iraola, M. Gabele, and G. Krieger, "First spaceborne demonstration of digital beamforming for azimuth ambiguity suppression," IEEE Trans. Geosci. Remote Sens., vol. 51, no. 1, pp. 579590, Jan. 2013.

[9] J. Mittermayer, S. Wollstadt, P. Prats, R. Scheiber, and W. Koppe, "Staring spotlight imaging with TerraSAR-X," in Proc. IEEE International Geoscience and Remote Sensing Symposium (IGARSS'12), Munich, Germany, Jul. 22-27, 2012.

[10] P. Prats-Iraola, R. Scheiber, M. Rodriguez-Cassola, S. Wollstadt, J. Mittermayer, B. Bräutigam, M. Schwerdt, A. Reigber, and A. Moreira, "High precision SAR focusing of Terrasar-X experimental staring spotlight data," in Proc. IEEE International Geoscience and Remote Sensing Symposium (IGARSS'12), Munich, Germany, Jul. 22-27, 2012.

[11] J. Mittermayer, S. Wollstadt, P. Prats, and R. Scheiber, "The TerraSAR$\mathrm{X}$ staring spotlight mode concept," IEEE Trans. Geosci. Remote Sens., 
2013.

[12] A. Meta, P. Hoogeboom, and L. P. Lighthart, "Signal processing for FMCW SAR," IEEE Trans. Geosci. Remote Sens., vol. 45, no. 11, pp. 3519-3532, Nov. 2007.

[13] A. Ribalta, "Time-domain reconstruction algorithms for FMCW-SAR," IEEE Geosci. Remote Sens. Lett., vol. 8, no. 3, pp. 396-400, 2011.

[14] K. Eldhuset, "A new fourth-order processing algorithm for spaceborne SAR," IEEE Trans. Aerosp. Electron. Syst., vol. 34, no. 3, pp. 824-835, 1998.

[15] D. D'Aria and A. Monti Guarnieri, "High-resolution spaceborne SAR focusing by SVD-Stolt," IEEE Geosci. Remote Sens. Lett., vol. 4, no. 4 , pp. 639-643, Oct. 2007.

[16] R. Bamler, F. Meyer, and W. Liebhart, "Processing of bistatic SAR data from quasi-stationary configurations," IEEE Trans. Geosci. Remote Sens., vol. 45, no. 11, pp. 3350-3358, Nov. 2007.

[17] A. Moreira and Y. Huang, "Airborne SAR processing of highly squinted data using a chirp scaling approach with integrated motion compensation," IEEE Trans. Geosci. Remote Sens., vol. 32, no. 5, pp. 1029-1040, Sep. 1994.

[18] A. Moreira, J. Mittermayer, and R. Scheiber, "Extended chirp scaling algorithm for air- and spaceborne SAR data processing in stripmap and ScanSAR imaging modes," IEEE Trans. Geosci. Remote Sens., vol. 34, no. 5, pp. 1123-1136, Sep. 1996.

[19] G. Fornaro, "Trajectory deviations in airborne SAR: Analysis and compensation," IEEE Trans. Aerosp. Electron. Syst., vol. 35, no. 3, pp. 997-1009, Jul. 1999.

[20] H. Breit, T. Fritz, U. Balss, M. Lachaise, A. Niedermeier, and M. Vonavka, "TerraSAR-X SAR processing and products," IEEE Trans. Geosci. Remote Sens., vol. 48, no. 2, pp. 727-740, Feb. 2010.

[21] J. C. Curlander and R. N. McDonough, Synthetic Aperture Radar: Systems and Signal Processing. New York, USA: John Wiley \& Sons, 1991, ch. 4.4.1 'Segmentation of the Correlator', pp. 159 - 164.

[22] J. Mittermayer, A. Moreira, and O. Loffeld, "Spotlight SAR data processing using the frequency scaling algorithm," IEEE Trans. Geosci. Remote Sens., vol. 37, no. 5, pp. 2198-2214, Sep. 1999.

[23] J. Mittermayer, R. Lord, and E. Boerner, "Sliding spotlight SAR processing for TerraSAR-X using a new formulation of the extended chirp scaling algorithm," in Proc. IEEE International Geoscience and Remote Sensing Symposium (IGARSS'03), vol. 3, Toulouse, France, Jul. 21-25, 2003, pp. 1462-1464.

[24] R. Lanari, M. Tesauro, E. Sansosti, and G. Fornaro, "Spotlight SAR data focusing based on a two-step processing approach," IEEE Trans. Geosci. Remote Sens., vol. 39, no. 9, pp. 1993-2004, Sep. 2001.

[25] G. Fornaro, G. Franceschetti, and S. Perna, "On center-beam approximation in SAR motion compensation," IEEE Geosci. Remote Sens. Lett., vol. 3, no. 2, pp. 276-280, Apr. 2006.

[26] Y. T. Yoon, M. Eineder, N. Yague-Martinez, and O. Montenbruck, "TerraSAR-X precise trajectory estimation and quality assessment," IEEE Trans. Geosci. Remote Sens., vol. 47, no. 6, pp. 1859-1868, Jan. 2009.

[27] S. N. Madsen, "Motion compensation for ultra wide band SAR," in Proc. IEEE International Geoscience and Remote Sensing Symposium (IGARSS'01), vol. 3, Sydney, Australia, Jul. 9-13, 2001, pp. 1436-1438.

[28] G. Fornaro, G. Franceschetti, and S. Perna, "Motion compensation of squinted airborne SAR raw data: Role of processing geometry," in Proc. IEEE International Geoscience and Remote Sensing Symposium (IGARSS'04), vol. 2, Anchorage, Alaska, USA, Sep. 20-24 2004, pp. $1518-1521$.

[29] A. Reigber, E. Alivizatos, A. Potsis, and A. Moreira, "Extended wavenumber domain SAR focusing with integrated motion compensation," IEE Proc. Radar Sonar Navig., vol. 153, no. 3, pp. 301-310, Jun. 2006.

[30] A. Reigber, R. Scheiber, M. Jäger, P. Prats-Iraola, I. Hajnsek, T. Jagdhuber, K. P. Papathanassiou, M. Nannini, E. Aguilera, S. Baumgartner, R. Horn, A. Nottensteiner, and A. Moreira, "Very-high-resolution airborne synthetic aperture radar imaging: Signal processing and applications," Proceedings of the IEEE, vol. 101, no. 3, pp. 784-797, Mar. 2013.

[31] H. M. J. Cantalloube and C. E. Nahum, "Airborne SAR-efficient signal processing for very high resolution," Proceedings of the IEEE, no. 3 , pp. 784-797, Mar. 2013.

[32] M. Jehle, D. Perler, D. Small, A. Schubert, and E. Meier, "Estimation of atmospheric path delays in TerraSAR-X data using models vs. measurements," Sensors, vol. 8, no. 12, pp. 8479-8491, 2008.

[33] M. Eineder, C. Minet, P. Steigenberger, X. Cong, and T. Fritz, "Imaging geodesy-toward centimeter-level ranging accuracy with TerraSAR-X," IEEE Trans. Geosci. Remote Sens., vol. 49, no. 2, pp. 661-671, 2011.
[34] J. Boehm, A. Niell, P. Tregoning, and H. Schuh, "Global mapping function (gmf): A new empirical mapping function based on numerical weather model data," Geophysical Research Letters, vol. 33, no. 7, p. L07304, 2006.

[35] F. Rocca, "Modeling interferogram stacks," Geoscience and Remote Sensing, IEEE Transactions on, vol. 45, no. 10, pp. 3289-3299, 2007.

[36] R. K. Raney, H. Runge, R. Bamler, I. Cumming, and F. H. Wong, "Precision SAR processing using chirp scaling," IEEE Trans. Geosci. Remote Sens., vol. 32, no. 4, pp. 786-799, Jul. 1994.

[37] G. W. Davidson and I. Cumming, "Signal properties of spaceborne squint-mode SAR," IEEE Trans. Geosci. Remote Sens., vol. 35, no. 3, pp. 611-617, May 1997.

[38] S. Wollstadt, P. Prats, M. Bachmann, J. Mittermayer, and R. Scheiber, "Scalloping correction in TOPS imaging mode SAR data," Geoscience and Remote Sensing Letters, IEEE, vol. 9, no. 4, pp. 614-618, 2012.

[39] B. Grafmuller, A. Herschlein, and C. Fischer, "The TerraSAR-X antenna system," in Radar Conference, 2005 IEEE International, 2005, pp. 222 225.

[40] U. Balss, H. Breit, and T. Fritz, "Noise-related radiometeric correction in the TerraSAR-X multimode SAR processor," IEEE Trans. Geosci. Remote Sens., vol. 48, no. 2, pp. 741-750, Feb. 2010.

[41] T. Kempf, H. Anglberger, and H. Suess, "Depth-of-focus issues on spaceborne very high resolution SAR," in Proc. IEEE International Geoscience and Remote Sensing Symposium (IGARSS), Munich, Germany, 2012, pp. 7448-7451.

[42] M. Rodriguez-Cassola, P. Prats-Iraola, F. De Zan, R. Scheiber, and A. Reigber, "Doppler-related focusing aspects in the TOPS imaging mode," in Proc. IEEE International Geoscience and Remote Sensing Symposium (IGARSS), Melbourne, Australia, Jul. 2013.

[43] K. A. C. de Macedo and R. Scheiber, "Precise topography- and aperturedependent motion compensation for airborne SAR," IEEE Geosci. Remote Sens. Lett., vol. 2, no. 2, pp. 172-176, Apr. 2005.

[44] P. Prats, K. A. C. de Macedo, A. Reigber, R. Scheiber, and J. J. Mallorqui, "Comparison of topography- and aperture dependent motion compensation algorithms for airborne SAR," IEEE Geosci. Remote Sens. Lett., vol. 4, no. 3, pp. 349-353, Jul. 2007.

[45] I. G. Cumming and F. H. Wong, Digital Processing of Synthetic Aperture Radar Data. Algorithms and Implementation. Boston, London: Artech House, 2005.

[46] R. Lanari, "A new method for the compensation of the SAR range cell migration based on the chirp Z-transform," Geoscience and Remote Sensing, IEEE Transactions on, vol. 33, no. 5, pp. 1296-1299, 1995.

[47] C. Cafforio, C. Prati, and F. Rocca, "SAR data focusing using seismic migration techniques," IEEE Trans. Aerosp. Electron. Syst., vol. 27, no. 2, pp. 194-207, Mar. 1991.

[48] M. Vandewal, R. Speck, and H. Süß, "Efficient and precise processing for squinted spotlight SAR through a modified Stolt mapping," EURASIP Journal on Applied Signal Processing, vol. 2007, no. 1, pp. 33-33, 2007.

[49] J. Janoth, S. Gantert, T. Schrage, and A. Kaptein, "TerraSAR next generation - mission capabilities," in Proc. IEEE International Geoscience and Remote Sensing Symposium (IGARSS'13), Melbourne, Australia, Jul. 22-26, 2013

[50] M. Rodriguez-Cassola, P. Prats-Iraola, A. Reigber, and A. Moreira, "Estimation of troposheric delays using synthetic aperture radar and squint diversity," in Proc. IEEE International Geoscience and Remote Sensing Symposium (IGARSS), Melbourne, Australia, Jul. 2013. 


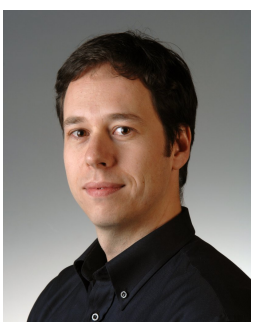

Pau Prats-Iraola (S'03-M'06-SM'13) was born in Madrid, Spain, in 1977. He received the Ingeniero degree in telecommunication engineering and the $\mathrm{Ph} . \mathrm{D}$. degree from the Universitat Politècnica de Catalunya (UPC), Barcelona, Spain, in 2001 and 2006, respectively.

In 2001, he was a Research Assistant at the Institute of Geomatics, Spain. In 2002, he was at the Department of Signal Theory and Communications, UPC, where he worked in the field of airborne repeat-pass interferometry and airborne differential SAR interferometry. From December 2002 to August 2006, he was an Assistant Professor at the Department of Telecommunications and Systems Engineering, Universitat Autònoma de Barcelona, Barcelona, Spain. In 2006, he joined the Microwaves and Radar Institute, German Aerospace Center (DLR), Wessling, Germany, where, since 2009, he has been the Head of the Multimodal Algorithms Group. His research interests include high-resolution airborne/spaceborne SAR processing and SAR interferometry.

Dr. Prats-Iraola was the recipient of the first prize of the Student Paper Competition of the 2005 IEEE International Geoscience and Remote Sensing Symposium (IGARSS), Seoul, Korea. In 2012, he was awarded with the DLR Science Award

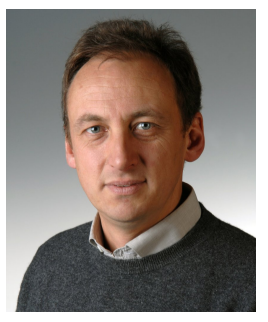

Rolf Scheiber received the Diploma degree in electrical engineering from the Technical University of Munich, Germany in 1994. In 2003 he obtained the $\mathrm{PhD}$ degree also in electrical engineering from the University of Karlsruhe, Germany with a thesis on airborne SAR interferometry.

Since 1994 he is with the German Aerospace Center (DLR), Microwaves and Radar Institute, where he developed the operational high precision interferometric SAR processor for its E-SAR airborne sensor. Since 2001 he is heading the SAR signal processing group within the SAR technology department. His current research interests include algorithm development for high resolution air- and spaceborne SAR focusing, SAR interferometry, differential SAR interferometry, SAR tomography as well as radio sounding algorithms and applications.

Dr. Scheiber was awarded as co-author with the 1996 GRSS Transactions Prize Paper Award for the contribution "Extended Chirp Scaling Algorithm for Air- and Spaceborne SAR Data Processing in Stripmap and ScanSAR Imaging Modes" in 1997

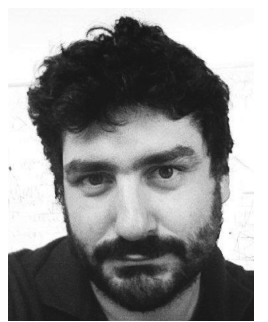

Marc Rodriguez-Cassola was born in Barcelona, Spain, in 1977. He received the Ingeniero degree in telecommunication engineering from Universidad Publica de Navarra, Pamplona, Spain, in 2000, and the Dr.-Ing. degree in electrical engineering from the Karlsruhe Institute of Technology, Germany, in 2012.

From 2000 to 2001, he was a radar hardware engineer at CETP/CNRS, Saint Maur des Fosses, France. From 2001 to 2003, he worked as a software engineer at Altran Consulting in Germany. Since 2003, he has been with the Microwaves and Radar Institute, German Aerospace Center (DLR), Germany, where he has been working on airborne and spaceborne SAR system analysis and data processing. His current research interests encompass radar signal processing, innovative high-precision SAR imaging algorithms, and radar system analysis and applications.

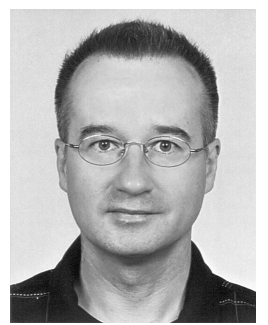

Josef Mittermayer was born in Wartenberg, Oberbayern, Germany, in 1967. He received the Diploma in electrical engineering from the Technical University of Munich, Munich, Germany, in 1995, with a thesis on ScanSAR processing, the M.S. degree in space system engineering from the Delft University of Technology, Delft, The Netherlands, in 2004, and the Ph.D. degree from the University of Siegen, Siegen, Germany, in 2000, in the field of spotlightSAR processing.

From 1994 to 2001, he was with the Signal Processing Group, Microwaves and Radar Institute, German Aerospace Center (DLR), Bavaria, Germany. From 2002 to 2008, he was working in the TerraSAR-X project with DLR. From January 2004 until the end of the commissioning phase in 2008, he was the Group Leader and the Project Manager of System Engineering and Calibration, one of the three subprojects that form the TerraSAR-X Ground Segment. In addition, he was technically responsible for the TerraSAR-X commissioning phase. He is currently as Scientist with the Microwaves and Radar Institute, working in the field of SAR system engineering and SAR processing.

Dr. Mittermayer and his colleagues were recipients of the IEEE Geoscience and Remote Sensing Society Transactions Prize Paper Award for a paper on airand spaceborne stripmap and ScanSAR processing in 1996. In 2001, he was a recipient of the DLR Science Award for his work on spotlight-SAR processing. In 2011, he was a recipient of the DLR Forschungssemester for his contribution to the TerraSAR-X project.

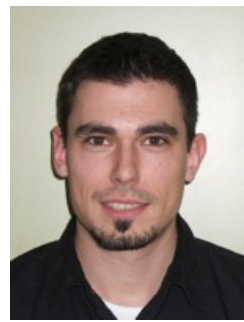

Steffen Wollstadt received the Dipl.-Ing. (M.Sc.) degree in electrical engineering from the Technical University of Darmstadt, Darmstadt, Germany, in 2005 , with a thesis on metamaterial-based microstrip antenna design.

Since 2006, he has been with the Microwaves and Radar Institute, German Aerospace Center (DLR), Bavaria, Germany. From 2006 to 2008, he was a Project Engineer with the Satellite Synthetic Aperture Radar (SAR) Systems department in the System Engineering and Calibration part of the TerraSAR$\mathrm{X}$ ground segment, where he worked on the TerraSAR-X and TanDEM-X instrument command generation. Since 2009, he has been with the Radar Concepts Department, Microwaves and Radar Institute, where he has been working on Terrain Observation by Progressive Scans (TOPS) Sentinel-1 image quality and SAR performance investigations. His current main interests focus on experiments with TerraSAR-X and TanDEM-X about novel imaging modes, particularly focusing on interferometric problems.

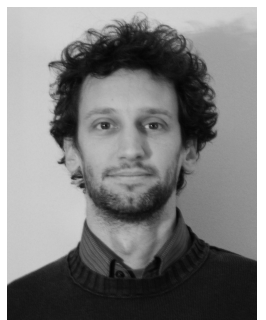

Francesco De Zan received the Master's degree in telecommunication engineering and the Ph.D. degree with a thesis on SAR interferometry and decorrelating targets from Politecnico di Milano, Milan, Italy, in 2004 and 2008, respectively.

During his studies he has collaborated with T.R.E. Milan, on persistent scatterer interferometry and with ESA about the Interferometric Wide Swath Mode of the Sentinel-1 mission. In 2007, he visited for six months the Stanford Exploration Project at Stanford University, Stanford, CA, USA. He has been with the Microwaves and Radar Institute of DLR (German Aerospace Center), Oberpfaffenhofen, Germany, since 2008. His current research interests include mission design and performance analysis of future interferometric SAR missions (e.g. BIOMASS, Tandem-L), interferometric/radargrammetric calibration of TanDEM-X, modeling and performance analysis for SAR interferometry and speckle tracking. 


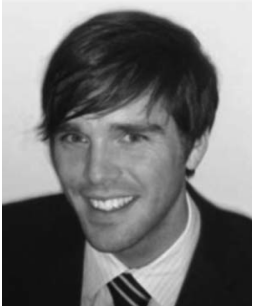

Benjamin Bräutigam received the Dipl.Ing. degree in electrical engineering from the Universität Karlsruhe (TH), Karlsruhe, Germany, in 2003.

In 2003, he was a Visiting Scientist at the Environmental Technology Laboratory, National Oceanic and Atmospheric Administration, Boulder, CO. In 2004, he joined the Microwaves and Radar Institute, German Aerospace Center (DLR), Wessling, Germany. He was responsible for internal instrument calibration in spaceborne synthetic aperture radar projects like TerraSAR-X, TanDEM-X, and Sentinel-1. Since 2008, he has been the Head of the System Performance Group, Microwaves and Radar Institute, DLR, working on TerraSAR-X and TanDEM-X. His major research interests include the development and analysis of innovative methods for instrument calibration and performance monitoring.

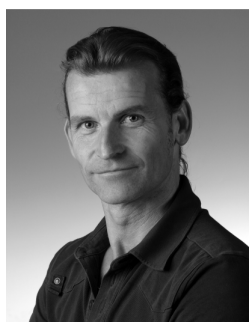

Marco Schwerdt received the Dipl.-Ing degree in electrical engineering and the Dr.-Ing. degree, with a thesis on electrooptical E-field sensors, from the Technical University of Berlin, Berlin, Germany.

Since 1998, he has been with the Microwaves and Radar Institute, German Aerospace Center (DLR), Oberpfaffenhofen, Germany, where he is working on synthetic aperture radar (SAR) calibration methods and performance analysis tools. Since 2000, he has been the Head of the Radar Calibration Group, performing various radar calibration activities for different SAR missions like X-band Synthetic Aperture Radar on the Shuttle Radar Topography Mission or the ScanSAR mode of Advanced Synthetic Aperture Radar (ASAR)/Envisat. He was responsible for the successful calibration of the German TerraSAR-X and TanDEM-X satellites, launched in 2007 and 2010, respectively. Furthermore, as part of the Global Monitoring Environment and Security Program, he is responsible for developing the overall SAR system calibration and validation plan for European Space Agency's Sentinel1 mission. Under his leadership, the DLR's comprehensive radar calibration facilities, including novel tools for product quality control and performance analysis, have been maintained and extended. His major research interest includes the development of innovative and efficient calibration methods. Dr Schwerdt was a recipient of the Der Deutsche Gründerfonds award in 1997 for establishing an enterprise of manufacturing electrooptical field sensors under the patronage of the German Federal Minister for Science and Research.

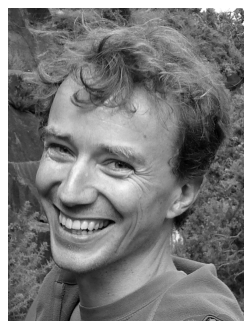

Andreas Reigber (M'02-SM'10) was born in $\mathrm{Mu}-$ nich, Germany, in 1970. He received the diploma degree in physics from the University of Constance, Germany, in 1997, the Ph.D. degree from the University of Stuttgart, Germany, in 2001, and the habilitation from the Berlin University of Technology, Germany, in 2008.

From 1996 to 2000, he has been with the Microwave and Radar Institute of the German Aerospace Center (DLR), Oberpfaffenhofen, Germany, working in the field of polarimetric SAR tomography. In 2001, he joined the Antenna, Radar and Telecom laboratories of the University of Rennes 1, Rennes, France, for a postdoc on radar polarimetry and polarimetric interferometry. From 2002 to 2007, he has been research associate at the Computer Vision and Remote Sensing laboratories of the Berlin University of Technology, Germany. Since 2008 he is back at the DLR Microwave and Radar Institute, where he is now head of the SAR technology department and directing the airborne SAR activities of the institute. His current main research interests are the various aspects of multimodal SAR, like SAR interferometry, SAR polarimetry, SAR tomography and time-frequency analyses, as well as filtering and classification aspects of high-resolution SAR data.

Dr. Reigber has received the EUSAR 2000 Student Prize Paper Award for an article on SAR remote sensing of forests, the IEEE TGRS Transactions Prize Paper Award in 2001 for a work on polarimetric SAR tomography as well as the IEEE TGRS Letters Prize Paper Award in 2006 for a work on multipass SAR processing.

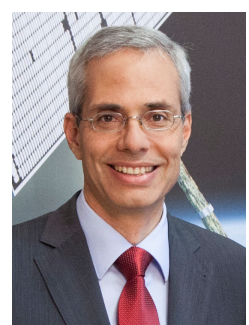

Alberto Moreira (M'92-S'96-F'04) received the B.S.E.E. and M.S.E.E. degrees from the Aeronautical Technological Institute (ITA), São José dos Campos, in 1984 and 1986, respectively, and the Eng. Dr. degree (with honors) from the Technical University of Munich, Germany, in 1993.

From 1996 to 2001, he was the Chief Scientist and Engineer with the SAR Technology Department, German Aerospace Center (DLR), Oberpfaffenhofen, Germany. Under his leadership, the DLR airborne SAR system has been upgraded to operate in innovative imaging modes like polarimetric SAR interferometry and SAR tomography. Since 2001, he has been the Director of the Microwaves and Radar Institute at DLR. The Institute contributes to several scientific programs and space projects for actual and future airborne and spaceborne SAR missions like TerraSAR-X, TanDEM-X, PAZ, Sentinel-1, SAR-Lupe, BIOMASS and Tandem-L. The mission TanDEM-X, led by his Institute, has successfully started the operational phase in December 2010. Prof. Moreira is the initiator and Principal Investigator for this mission. Since 2003, he is also a Full Professor with the Karlsruhe Institute of Technology, Germany, in the field of microwave remote sensing. He has more than 350 publications in international conferences and journals and is the holder of 15 patents in the radar and antenna field. His professional interests and research areas encompass radar end-to-end system design and analysis, innovative microwave techniques and system concepts, signal processing, and remote sensing applications.

$\mathrm{He}$ is a member of the IEEE Geoscience and Remote Sensing Society (GRSS) Administrative Committee (1999-2001, 2004-2013, 2010 as President, 2011-2013 as Past-President), was the Founder and Chair of the GRSS German Chapter (2003-2008) and Associate Editor for the IEEE GRS Letters (2003-2007) and for the IEEE TGRS (since 2005). He and his colleagues received the GRS-S Transactions Prize Paper Awards in 1997, 2001, and 2007, the IEEE W.R.G. Baker Award in 2012 from the IEEE Board of Directors and were nominated for the Federal Prize for Innovation and Future Technologies of the German President (2012). He is also recipient of the DLR Science Award (1995), IEEE Nathanson Award for the Young Radar Engineer of the Year (1999) and the IEEE Kiyo Tomiyasu Field Award (2007). He served as a member of the Board of Directors of the Information Technology Society of the German Association for Electrical, Electronic and Information Technologies (2003-2008), as chair of the Scientific and Technical Council of DLR (2009-2011), as chair of the DLR's Center of Excellence on Synthetic Aperture Radar (2000-2012) and is currently chairing the Helmholtz Alliance "Remote Sensing and Earth System Dynamic". He has contributed to the successful series of the European SAR conferences (EUSAR) since 1996 as a member of the Technical Program Committee, Technical Chairman (2000), Awards Chairman (2002-2004), General Chairman (2006), and Co-Chairman (2008) and has served as General Co-Chair for IGARSS'12 in Munich. 